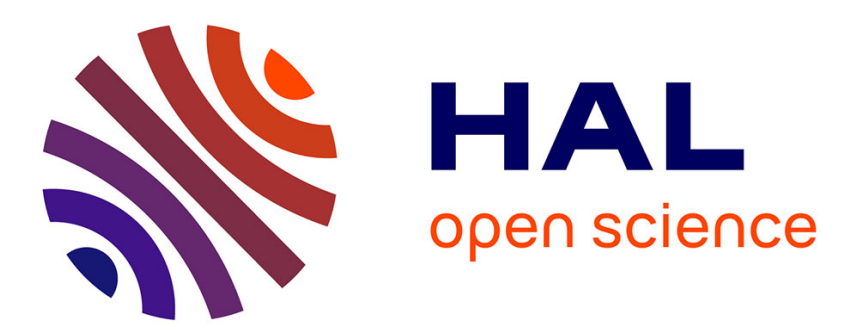

\title{
A low-order reduced model for the long range propagation of infrasound in the atmosphere
}

Michaël Bertin, Christophe Millet, Daniel Bouche

\section{To cite this version:}

Michaël Bertin, Christophe Millet, Daniel Bouche. A low-order reduced model for the long range propagation of infrasound in the atmosphere. Journal of the Acoustical Society of America, 2014, 136, pp.37-52. 10.1121/1.4883388 . cea-02106560

\section{HAL Id: cea-02106560 https://hal-cea.archives-ouvertes.fr/cea-02106560}

Submitted on 23 Apr 2019

HAL is a multi-disciplinary open access archive for the deposit and dissemination of scientific research documents, whether they are published or not. The documents may come from teaching and research institutions in France or abroad, or from public or private research centers.
L'archive ouverte pluridisciplinaire HAL, est destinée au dépôt et à la diffusion de documents scientifiques de niveau recherche, publiés ou non, émanant des établissements d'enseignement et de recherche français ou étrangers, des laboratoires publics ou privés. 
A low-order reduced model for the long range propagation of infrasound in the atmosphere

CEA, DAM, DIF, 91297 Arpajon, France

\footnotetext{
${ }^{1}$ e-mail: christophe.millet@cea.fr
} 


\section{Abstract}

This paper considers a class of low-order, range-dependent propagation models obtained 7 from the normal mode decomposition of infrasounds in complex atmospheres. The classical 8 normal mode method requires calculating eigenvalues for large matrices making the compu9 tation expensive, even though some modes have little influence on the numerically obtained 10 results. By decomposing atmospheric perturbations into a wavelet basis, it is shown that 11 the most sensitive eigenvalues provide the best reduced model for infrasound propagation. 12 These eigenvalues lie on specific curves in the complex plane that can be directly deduced 13 from atmospheric data through a WKB approach. The computation cost can be reduced 14 by computing the invariant subspace associated with the most sensitive eigenvalues. The 15 reduction method is illustrated in the case of the Fukushima explosion (12 March 2011). The implicitly-restarted Arnoldi algorithm is used to compute the 3 most sensitive modes 17 and the correct tropospheric arrival is found with a cost of $2 \%$ of the total run time. The cost can be further reduced by using a stationary phase technique. Finally, it is shown that 19 adding uncertainties triggers a stratospheric arrival even though the classical criteria, based 20 on the ratio of stratospheric sound speed to that at ground level, is not satisfied. 
Bertin, Millet and Bouche, J. of the Acoustical Society of America, p. 3

\section{Introduction}

The usual approach in studying infrasound propagation involves computing the acoustic component superimposed to a given atmospheric state, which is classically obtained from global empirical models (Drob et al. ${ }^{16}$, Hedin et al. ${ }^{20}$ ) as well as operational numerical weather prediction centers (e.g., NOAA global forecast system or ECMWF integrated forecast system). The justification of such an approach is questionable given that unresolved large-scale gravity waves, turbulence and matching conditions of the various available data may affect the waveforms in such a way that some arrivals may literally appear/disappear.

While a significant amount of data has become available on the temperature and wind structure of the atmosphere, it is not clear whether classical propagation techniques (ray tracing, PE method) are able to statistically capture the effects of small-scale uncertainties (Kulichkov et al. ${ }^{25}$, Chunchuzov et al. ${ }^{13}$, Hedlin et al. $\left.{ }^{21}\right)$.

The lack of knowledge concerning the atmosphere leads to consider the atmospheric structures as being generated by random gravity wave sources, an approach that was recently popularized by Drob et al. ${ }^{17}$. As far as statistics are concerned, the question of the computational cost is critical. According to the law of large numbers, the more runs we make, the closer the sample mean of the results should be to the true mean. This law, however, does not describe how the convergence takes place. Indeed, the number of simulation runs necessary to attain a given confidence level depends on random atmospheric structures 
Bertin, Millet and Bouche, J. of the Acoustical Society of America, p. 4

40

41

42

43

and thus, a statistical analysis may be computationally expensive.

This paper considers a class of low order propagation models obtained from the normal mode decomposition of infrasounds in complex atmospheres. While the ray theory cannot predict some important refracted paths (Waxler, Gilbert and Talmadge ${ }^{41}$ ), the normal mode technique can be used to simulate signals within environments that cover a wide range of vertical scales. The complete acoustic field is constructed by summing up contributions of modes, which can be obtained by seeking the roots of the so-called dispersion relation. In most cases the poles are complex, and thereby requires the use of a two-dimensional search algorithm. In addition to the difficulties inherent in searching in two dimensions, it is important to point out that the wave equation in a finite domain has an infinite set of discrete eigenvalues and thus, the number of numerically obtained modes is proportional to the number of grid points used. Then, for a fixed number of grid points per wavelength, the numerical cost increases with the frequency. Subsequently, Waxler ${ }^{39}$ has emphasized that the imaginary parts (the attenuation rates) can be obtained through a perturbative approach, by solving a fixed point equation with a Newton-like method. Such an approach, however, is restricted to poles that are close to the real axis. In this paper we are interested in the manner in which the modes provide the best reduced model (finding the dominant modes) and more generally we are interested in the behavior of the selected poles in the complex plane as the background atmospheric state is varied. 
Bertin, Millet and Bouche, J. of the Acoustical Society of America, p. 5

This work is an outgrowth of the authors' effort to find the simplest mathematical model in order to estimate signal statistics. It is often the case that first-principles modeling or system identification result in unnecessarily high-dimensional mathematical models. Model (order) reduction is about systematic approximation of such models. There are many advantages in working with models with low-dimensional state space since low-dimensional models are often easier to analyze and much faster to simulate. As pointed out by Antoulas ${ }^{3}$ in his survey on reduction methods, we can identify two sets of methods which are currently in use, namely the SVD (Singular Value Decomposition) based methods and the moment matching methods.

In fluid dynamics applications, SVD based methods are widely used, the most common approach being the Proper Orthogonal Decomposition (POD) method and its variants such as the BPOD (Balanced Proper Orthogonal Decomposition) (Rowley ${ }^{34}$ ). POD methods are based on the Galerkin projection of the dynamical model on a subspace obtained with a SVD analysis of experimental or numerical datasets. It is known that only a few POD modes can summarize the flow organization in many cases (Delville et al. ${ }^{15}$, Galletti ${ }^{18}$ ). It also appears that reduced-order models generally lose accuracy for flow parameters different from those used to generate the POD basis, even if a local improvement can be made with a sensitivity analysis of the modes $\left(H_{a y}{ }^{19}\right)$. For control optimization problems, instead of using a POD basis, some authors directly use the global eigenmodes as a projection basis, 
Bertin, Millet and Bouche, J. of the Acoustical Society of America, p. 6

even if it appears that it does not show sufficient robustness $\left(\right.$ Barbagallo $\left.^{5}\right)$. For instance, the optimal growth in a separated boundary-layer flow can be studied with the sum of the non-normal global modes (that are the most sensitive to any flow perturbation) (Akervik $\left.{ }^{2}\right)$.

The other set of methods, the moment matching approach, is not commonly used for fluid dynamics model reduction and is mostly found in structural dynamics or circuit simulations. It consists in using Krylov subspace methods to get a low-order model that shows a good approximation of the transfer function, and more precisely of the first coefficients of its Laurent expansion $\left(\right.$ Bai $^{4}$, Srinivasan Puri $\left.{ }^{36}\right)$. Arnoldi and Lanczos algorithms that perform iterative projections on Krylov subspaces $\left(\right.$ Watkins $^{38}$ ) are modified in order to guide the reduction to the best approximation of the transfer function. Progress in other Krylov subspace techniques allow the reduction of a large variety of dynamical systems, including second-order systems (Salimbahrami ${ }^{35}$ ) and nonlinear systems $\left(\right.$ Bai $\left.^{4}\right)$. With regards to the wave equation, it is easy to apply the Krylov-Arnoldi algorithm to compute a few of the smallest or largest modes of a large matrix obtained from the discretized problem. But there is no universal way in which the modes may be sorted, and indeed much of the emphasis in the present work is to show that the modes lie in the vicinity of regions in the complex plane, for each of which there are different features in the vertical sound speed profile.

The paper is organized as follows: the derivation of the range-dependent normal-mode method is described in section 2. A sensitivity analysis is discussed in section 3 and it is shown 
Bertin, Millet and Bouche, J. of the Acoustical Society of America, p. 7

97

that, to a first approximation, the most sensitive modes to waveguide perturbations provide the best low-order reduced model for long-range infrasound propagation. In section 4, we use a WKB approach to find the most sensitive regions in the complex plane and an implicitly restarted Arnoldi procedure is used to obtain the most sensitive modes. We show that the contribution of each mode is confined to a narrow range of frequencies so that when the stationary phase method is limited to the first most sensitive mode, it gives a very good approximation of the waveform. We illustrate this in the case of the Fukushima explosion that was detected at the Japanese infrasound station I30JP (12 March 2011), located $243 \mathrm{~km}$ away from the source. Finally, in section 5, we show that adding stratospheric uncertainties may trigger a stratospheric arrival even though the classical criteria, based on the ratio of stratospheric sound speed to that at ground level, is not satisfied.

\section{Normal mode decomposition of the pressure field}

\section{A. The mean atmosphere}

There is no consensus on how mean atmospheric specifications can be obtained from operational numerical weather predictions or atmospheric climate reanalysis. In this work, the wind and temperature profiles are extracted from the atmospheric database provided by the European Centre for Medium-Range Weather Forecasts (ECMWF). Following Millet et $a l .{ }^{30}$, the profiles are obtained from the ERA-Interim and 91-level datasets for altitudes less 
Bertin, Millet and Bouche, J. of the Acoustical Society of America, p. 8

than $50 \mathrm{~km}$ and $80 \mathrm{~km}$, respectively, and matched to statistical data for higher altitudes. The statistical data is obtained using empirical reference models, known as HWM-93 (Horizontal Wind Model) and MSIS-90 (Mass Spectrometer and Incoherent Radar Model). Although these models represent a compromise between the original data sources, they are known to present systematic differences, particularly near the mesopause (Hedin et al. ${ }^{20}$ ).

In this study, we consider the explosion of the Fukushima Daiichi nuclear power plant (suspected to be caused by hydrogen gas) that occurred on 12 March 2011. The recorded infrasound signal at the Japanese station I30JP is shown in figure 1 as well as the effective sound speed profiles. The profiles obtained from the 91-level dataset and the ERA-interim dataset are shown in figure 1, above the source and above the infrasound station I30JP. Recent simulations by Bertin et al. ${ }^{9}$ have shown that the signal is composed of two arrivals: a strong tropospheric arrival, 12-13 minutes after the explosion, followed by a weaker stratospheric 'tail'. As part of their study, Bertin et al. ${ }^{9}$ have suggested on numerical grounds that, due to the vertical profile between the source and the infrasound station, the amplitude of the first arrival can change by several orders of magnitude. It is therefore reasonable to conclude that the range-independent assumption breaks down for this tropospheric arrival, even though the waveform shape does not seem to be affected. In the present work, due to its strong dependence to the sound speed profile, we will first focus on the first arrival using a range-dependent approach. 
Bertin, Millet and Bouche, J. of the Acoustical Society of America, p. 9

It is not clear how small changes in the profile along the source-to-receiver path affect the infrasound propagation. The atmosphere is usually described as a thin layer of fluid in which any vertical structure has its counterpart in the horizontal direction. Following the method of multiple scales, the effective sound speed may be represented analytically in the form of the function $c(z, \epsilon r)$, where $r$ and $z$ are the distance from the source and altitude, respectively. Numerically, $\epsilon$ is a small parameter that can be interpreted either as a measure of a 'slow' coordinate $\epsilon r$ (Bender and $\operatorname{Orszag}^{8}$ ) or a perturbation of a given profile. The first order Taylor series expansion of $c$ about $\epsilon=0$ can be written as

$$
c(z, \epsilon r) \simeq c_{0}(z)+\epsilon c_{1}(z)
$$

where $c_{0}(z)=c(z, 0)$ is the profile above the source. Hence, the degree of horizontal inhomogeneity of the atmosphere can be considered as a perturbation. Here, $\epsilon$ will be deemed a small parameter and will be used as such later.

\section{B. The vertical structure equation}

As our starting point, we use the wave equation in cylindrical coordinates $(r, \theta, z)$.

The acoustic wave is assumed to be initiated by a ground-based localized excitation of frequency $\omega$.

The most elementary approach consists in entirely neglecting the horizontal variability of the medium: if the atmosphere is considered as uniform in the horizontal direction, the 
Bertin, Millet and Bouche, J. of the Acoustical Society of America, p. 10

151

vertical structure $\phi(z)$ of the pressure field satisfies the Helmholtz equation

$$
\frac{\mathrm{d}^{2} \phi}{\mathrm{d} z^{2}}+\left[\frac{\omega^{2}}{c^{2}(z)}-k^{2}\right] \phi=-\frac{\delta(z)}{2 \pi},
$$

which, for unbounded atmospheres, requires a boundedness condition as $z \rightarrow \infty$. In the above equation $c(z)$ is the effective sound speed, $k$ is the horizontal wavenumber and $\delta$ is the Dirac delta function which mimics a point source at $z=0$.

In the present work, we use the effective sound speed approximation and substitute the moving fluid by a motionless fluid with the effective sound speed, as proposed by Rayleigh ${ }^{33}$. The density effect can easily be considered by defining $\phi=p / \sqrt{\bar{\rho}}$, where $p$ and $\bar{\rho}$ are the pressure and the mean density, respectively. This transformation allows to reduce the original Helmholtz equation to the normal mode equation (2), provided the acoustic wavelength is not too large. Indeed, upon substituting $\phi$ into the original Helmholtz equation when the density depends on altitude, we obtain an aditional term $\frac{1}{4} \phi H^{-2}$, where $H$ is the atmospheric height scale. This term, however, can be neglected for frequencies larger than $0.1 \mathrm{~Hz}$ and the pressure field can readily be obtained from $p=\phi \sqrt{\bar{\rho}}$.

The solution of (2) is, within a constant of proportionality, the Green's function of the wave equation. Following the classical approach (e.g. Jensen et al. ${ }^{23}$ ), the spatial part $p$ of the wave field can be obtained from the discrete-spectrum contribution, which is composed 
Bertin, Millet and Bouche, J. of the Acoustical Society of America, p. 11

167

$$
\begin{aligned}
& \text { of the sum of the residues evaluated at the (simple) roots of the dispersion relation } D(k, \omega) \\
& \qquad p(r, z ; \omega)=\frac{i}{2} \sum_{j=0}^{N} \frac{\phi_{j}(z ; \omega) \phi_{j}(0 ; \omega)}{\partial_{k} D\left(k_{j}(\omega), \omega\right)} k_{j}(\omega) \mathrm{H}_{0}^{(1)}\left(k_{j}(\omega) r\right),
\end{aligned}
$$

where $\mathrm{H}_{0}^{(1)}$ is the Hankel function of the first kind (Abramowitz and Stegun ${ }^{1}$ ) and $\phi_{j}$ is the eigenfunction associated with the spatial eigenvalue $k_{j}(\omega)$. Equation (3) can be simplified by properly scaling $\phi_{j}$. Taking

$$
\int_{0}^{\infty} \phi_{j}^{2}(z ; \omega) \mathrm{d} z=1
$$

a straightforward calculation ( Jensen et al. ${ }^{23}$ ) gives $\partial_{k} D\left(k_{j}, \omega\right)=2 k_{j}(\omega)$ so that the pressure field at ground level $(z=0)$ reads

$$
p(r, 0 ; \omega)=\frac{i}{4} \sum_{j=0}^{N} \phi_{j}^{2}(0 ; \omega) \mathrm{H}_{0}^{(1)}\left(k_{j}(\omega) r\right),
$$

where $N$ is the number of modes involved in waveguide propagation. Here we follow Waxler 39 and use the approximation $\sqrt{\bar{\rho}(0)}=1$ in the calculation of ground-based signals. Note that, for unbounded atmospheres, $\phi_{j}$ is in general non-analytic in $k$ and the spatial part $p(r, z ; \omega)$ involves a continuous-spectrum contribution that arises from the integral along the branch cuts. In practice, however, this contribution can be neglected, provided we are sufficiently far from the source ( Waxler $\left.^{40},{ }^{39}\right)$.

The poles are readily identified as the zeroes of $D(k, \omega)$, i.e. the spatial eigenvalues $k_{j}(\omega)$. Zeroes of $D(k, \omega)$ may also be sought in terms of temporal eigenvalues $\omega_{j}(k)$ when the wavenumber is assumed to be real. In this work, we use a boundary value (or implicit) 
Bertin, Millet and Bouche, J. of the Acoustical Society of America, p. 12

182

method for solving the eigenvalue problem. The wave equation (2) is reduced to a linear algebraic equation using a pseudo-spectral technique (Candelier et al. ${ }^{12},{ }^{11}$ ). The global eigenvalues are obtained by applying a QR algorithm to the generalized eigenvalue problem. While care must be taken to preclude spurious eigenvalues, it is worth mentioning that such an approach can be applied to problems in which the eigenvalue appears nonlinearly (Bridges and Morris $^{10}$ ) so that the absorption can be considered within the same numerical framework.

The leading behaviour of the temporal response is then obtained by applying the inverse Fourier transform to (5) to obtain

$$
p(r, 0 ; t) \sim \frac{i}{8 \pi} \sum_{j=0}^{N} \int_{F} \phi_{j}^{2}(0 ; \omega) \mathrm{H}_{0}^{(1)}\left(k_{j}(\omega) r\right) \mathrm{e}^{-i \omega t} \mathrm{~d} \omega,
$$

where the integration is performed along the path $F$ in the complex $\omega$-plane. One recognizes in expression (6) a wave packet composed of freely evolving spatial modes generated by a localized pulsed disturbance, at $r=0$. The contour cannot be chosen arbitrarily since both the convergence of (6) and the causality condition, $p(r, z ; t)=0$ for $t<0$, should be ensured. As pointed out by Batchelor et al. ${ }^{22}$, the contour $F$ has to be chosen to lie above all temporal eigenvalues $\omega_{j}(k)$ as $k$ travels along the real axis. Thus, to avoid confusion, the frequency parameter $\omega$ is treated as a Fourier-Laplace transform variable, $\omega=\omega_{r}+i \omega_{i}$, with a small positive imaginary part $\omega_{i} \ll 1$. For this reason, the eigenvalues $k_{j}(\omega)$ lie in the upper half of the complex $k$-plane, as shown in figure 2. Note that in figure 2 , we introduce the phase 
Bertin, Millet and Bouche, J. of the Acoustical Society of America, p. 13

velocity $c_{r}=\omega_{r} / k_{r}$, where $k_{r}$ is the real part of $k$.

Further insight into the nature of the modes can be gained by examining the manner in which they evolve along some path in the complex $\omega$-plane. For a fixed, real $\omega$, the problem (2) on a finite domain is self-adjoint and thus, it has a discrete spectrum of the form $k_{1}<k_{2}<\ldots<k_{N}$ and every eigenspace is one-dimensional. These eigenvalues are real-valued wavenumbers of the guided-wave modes of the medium. For a complex frequency, the eigenvalues $k_{j}(\omega)$ are continued in the complex $k$-plane: the larger the imaginary part of $\omega$ the stronger the attenuation (given by the imaginary part of $k$ ) of the guided-wave modes. The reason that these modes are decaying along the $r$-direction is directly tied to the causality requirement which forces the $F$-contour to be placed above the real axis. Thus, even though a medium would support growing (i.e. unstable) responses, its localized excitation by a source that grows sufficiently fast in time produce only decaying responses in space. In addition, the decaying rate depends on vertical inhomogeneities covered by the corresponding eigenfunction. A very interesting aspect of using a complex frequency is that the imaginary parts of eigenvalues $k_{j}(\omega)$ may be arranged so that the modes can be associated with specific regions in the atmosphere and thus, the problem can be projected onto the invariant subspace corresponding to a subset of these modes.

\section{Normal modes for range-dependent atmospheres}

Most environments of interest are spatially non uniform in the horizontal direction $r$. 
Bertin, Millet and Bouche, J. of the Acoustical Society of America, p. 14

The previous notions can then be taken to apply locally as long as the non-uniformities of the medium are small over a typical acoustic wavelength. Thus, the horizontal variation of the medium is characterized by a slow space variable $R=\epsilon r$, where $\epsilon$ is a small parameter of the same order of magnitude as the scaled nonuniformities. Decomposition into local normal modes leads to a dispersion relation of the form $D(k, \omega ; R)=0$. The eigenvalue problem is therefore solved for a discrete set of ranges $R_{1}, R_{2}, \ldots, R_{N}$.

In going from one range to another, we assume that the modes couple adiabatically, i.e. without any transfer of energy into other modes. This approximation, that was introduced in the study of underwater acoustics by Pierce $^{32}$, is a reasonable compromise between accuracy and run time. The WKB approximation then reads

$$
p(z ; \omega, R) \simeq \frac{i \mathrm{e}^{-i \pi / 4}}{2 \sqrt{2 \pi r}} \sum_{j=0}^{N} \frac{\phi_{j}(0 ; \omega, R) \phi_{j}(z ; \omega, R)}{\sqrt{k_{j}(\omega, R)}} \exp \left\{\frac{i}{\epsilon} \int_{0}^{R} k_{j}(\omega, s) \mathrm{d} s\right\}
$$

where $\phi_{j}(z ; \omega, R)$, which is the eigenfunction associated with the local wavenumber $k_{j}(\omega, R)$ (for a given $\omega$ ), satisfies the condition

$$
\int_{0}^{\infty} \phi_{j}(z ; \omega, R) \frac{\partial \phi_{j}}{\partial R}(z ; \omega, R) \mathrm{d} z=0
$$

where the derivative can be approximated through a finite difference.

Figure 2 shows the trajectories of the local eigenvalues $k_{j}$ as $R$ increases from the source $R=0$ to the infrasound station I30JP. While most eigenvalues are not significantly affected by horizontal variations of the vertical profile, the eigenvalues which are close to a cusp at 
Bertin, Millet and Bouche, J. of the Acoustical Society of America, p. 15

$c_{r} \simeq 335 \mathrm{~m} \cdot \mathrm{s}^{-1}$ trace the longest trajectories in the $k$-plane (essentially along the imaginary axis). Such eigenvalues are strongly sensitive to horizontal variations and correspond to waves travelling with exactly the phase velocity at a local maximum of $c(z)$ (which is also a double turning point in the limit $\omega \rightarrow \infty)$. This maximum is associated with a low altitude tropospherical waveguide (see figure 1), below $5 \mathrm{~km}$ altitude. Hence, waves of this kind strongly depend on large-scales, as a result of the weak coupling between horizontal and vertical low-altitude atmospheric structures, through the small parameter $\epsilon$. A closer examination of the trajectories in the complex $k$-plane shows that the path lengths of the most sensitive modes are smaller for the 91-level dataset (figures 2c,d). In spite of this, the phase velocity change (along the real axis) is larger and thus, the arrival time of the corresponding waveform is more affected.

The cusp may be exploited to track the most sensitive eigenvalues: the appearance of a cusp in the spectrum at a particular $c_{r}$ is an indication that the eigenvalues are on the verge of being critically dependent on small variations in the vertical profile. This criterion, which is completely general, may be used to reduce the complexity (and computation time) of any range-dependent infrasound propagation problem.

\section{Sensitivity of modes to the mean atmosphere}

This work focuses on the underlying assumption that the mean atmospheric state is fixed and is given by operational numerical weather predictions or atmospheric climate reanalysis. 
Bertin, Millet and Bouche, J. of the Acoustical Society of America, p. 16

Upon substitution of (9) into (10), we obtain

$$
\frac{\partial k_{j}}{\partial \epsilon}=\sum_{m} \sum_{n} c_{m n} K_{j m n}
$$

where each wavelet $\Psi_{m n}$ is generated by translation and dilatation of a function $\Psi_{m n}(z)=$ $a^{-m / 2} \Psi\left(a^{-m} z-n b\right)$, with $a>1$ and $b>0$, so that the basis depicts every position and every scale.

The sensitivity of the eigenvalue $k_{j}$ is obtained by substituting (1) into (2) and then differentiating with respect to $\epsilon$. Taking an inner product with the eigenfunction $\phi_{j}$ leads to

$$
\frac{\partial k_{j}}{\partial \epsilon}=-\frac{\omega^{2}}{k_{j}} \int_{0}^{\infty} \frac{\phi_{j}^{2}(z) c_{1}(z)}{c_{0}^{3}(z)} \mathrm{d} z
$$


Bertin, Millet and Bouche, J. of the Acoustical Society of America, p. 17

269

where $K_{j m n}$ is given by

$$
K_{j m n}=-\frac{\omega^{2}}{k_{j}} \int_{0}^{\infty} \frac{\phi_{j}^{2}(z)}{c_{0}^{2}(z)} \Psi_{m n}(z) \mathrm{d} z .
$$

It is clear from (12) that the components $K_{j m n}$ are nothing but the (complex) sensitivities of $k_{j}$ to each wavelet $\Psi_{m n}$. In practice, these components are easily computed through the multiresolution discrete wavelet transform of $\phi_{j}^{2} / c_{0}^{2}$ with the Mallat algorithm that only involves recursive filterings $\left(\right.$ Mallat $\left.^{28}\right)$.

Now, to obtain a relation between the sensitivity of a given mode and its relative contribution to the sum of the residues (5), we use the completeness and the orthonormality of the wavelet basis to deduce from (12) that

$$
\phi_{j}^{2}(z)=-\frac{k_{j} c_{0}^{2}(z)}{\omega^{2}} \sum_{m} \sum_{n} K_{j m n} \Psi_{m n}(z)
$$

27 Upon substituting (13) into (5), the ground-based pressure field becomes

$$
p(r, 0 ; \omega) \sim-\frac{i c_{0}(0)^{2}}{4 \pi} \sum_{j} \frac{k_{j}(\omega)}{\omega^{2}} \sum_{(m, n)} K_{j m n}(\omega) \mathrm{H}_{0}^{(1)}\left(k_{j}(\omega) r\right) \Psi_{m n}(0) .
$$

For a given mode, the above formula presents the advantage of determining which wavelets are relevant to the ground-based pressure field. We conclude, as expected intuitively, that only the wavelets that connect the perturbation at a given altitude to the source $\left(\Psi_{m n}(0) \neq 0\right)$ play a role in the sum (14). For these wavelets, the larger the sensitivity $K_{j m n}$, the larger the contribution of the wavelet to the signal. 
Bertin, Millet and Bouche, J. of the Acoustical Society of America, p. 18

Figure 3 gives the sensitivities $K_{j m n}$ of the three most sensitive modes to D10 wavelets (Daubechies ${ }^{14}$ ) as functions of the frequency $\omega_{r}$. The results are shown for the wavelet that maximizes the sensitivity, among 2046 wavelets that cover the altitudes in the range $0-120 \mathrm{~km}$ and the wavelengths from $117 \mathrm{~m}$ to $60 \mathrm{~km}$. As $\omega_{r}$ is increased, a subset of normal modes becomes highly sensitive to low altitude wavelets (figure 3c) and correspondingly their contribution to the ground-based pressure field increases, as shown in figure 4 . Indeed, for each mode, the sensitivity can be described by a few of the wavelets $(m, n)$ whose contributions are maximum over a narrow range of frequencies. These wavelets provide the vertical structures that are responsible for the transition from the cusp to a region of constant imaginary part, as shown in figure 3b. Note that the region where the most sensitive modes converge (as $\omega_{r} \rightarrow \infty$ ) can be obtained through a WKB analysis of $(2)$. The WKB modes are given by dashed lines in figure 3. This holds despite the fact that such an approximation fails to describe the transition.

It is important to point out that the family of (most sensitive) modes issuing from the cusp gives the leading-order contribution of (5) over frequencies as large as $1 \mathrm{~Hz}\left(\omega_{r}=2 \pi\right)$. Since the integration in $k$ is performed first, we observe that the integral can be replaced by the sum of contribution of three poles. Figure 4 shows the truncation of (5) to the third most sensitive modes as a function of $\omega_{r}$ and $z$, for $r=243 \mathrm{~km}$. The first of these modes gives the leading order behavior up to $0.5 \mathrm{~Hz}$ (figure 4a), whereas the third mode is required 
Bertin, Millet and Bouche, J. of the Acoustical Society of America, p. 19

as it describes the high frequency content close to the ground (figure 4c). Given that $\omega$ is a continuous variable, there are an infinite number of such contributions, which have to be included by the integration over $\omega$. However, if the Fourier transform of the source is zero outside a finite-length interval, usually taken to be $0 \leq \omega \leq \omega_{0}$, the contribution of high frequencies $\omega>\omega_{0}$ turns out to be zero, as a result of the convolution theorem.

The previous results can be interpreted within the setting of the range-dependent formulation presented in section II.C. Provided the sound speed profile is slowly varying in the horizontal direction, the expansion (1) is the first order Taylor series of $c(z)$ about $R=0$ and $\epsilon$ plays the same role as in the section II.C. When considering the acoustic propagation downstream, $k_{j}(R ; \omega)$ traces out a trajectory issuing from the cusp. The associated contribution as given by (5) becomes dominant over a frequency range that depends on vertical structures of $c_{1}(z)$ and thus, a lower bound for the frequency range can be obtained from the sensitivities. For each $R$, this lower bound plays the role of a cut-off frequency $\omega_{0}(R)$.

On the other hand, it can be shown that the Hankel function in (6) vanishes as $\omega_{r} \rightarrow \infty$.

\section{A low-order reduced model}

The basic principle in the reduction methods consists in computing a small number of relevant modes (a part of the spectrum) through specific methods. If a matrix is really large, the computation of its complete spectrum is out of reach. However, as noted in the previous section, we only require the computation of the few of the eigenvalues that are closest to 
Bertin, Millet and Bouche, J. of the Acoustical Society of America, p. 20

321 some specified target value. Eigenvalue methods for large problems are designed to perform tasks like these by computing the invariant subspace associated with the desired eigenvalues.

\section{A. A WKB analysis of the spectrum}

A close examination of figure 2 shows that the eigenvalues lie on interconnected curves that are related to specific regions in the effective sound speed profile. The global structure of these curves can be obtained using an analysis which is similar to that of Bender and Orszag ${ }^{8}$, except that the eigenvalues are complex. The main assumption, besides the exclusion of range-dependence, is that the frequency is sufficiently large, so that we have $K=\epsilon k=O(1)$ and $\Omega=\epsilon \omega=O(1)$, where $\epsilon$ is a small parameter. Using this approximation in (2) gives

$$
\epsilon^{2} \frac{d^{2} p}{d z^{2}}=Q(z) p
$$

where $Q$ is given by

$$
Q(z)=K^{2}-\frac{\Omega^{2}}{c^{2}(z)}
$$

For a real frequency, the WKB analysis performed in the limit $\epsilon \rightarrow 0$ for a vertical profile $c(z)$ with the typical shape displayed in figure 5 shows that the modes of the "bounded states" (i.e. with $K_{1}<K<K_{2}$ ) are given by the well-known Bohr-Sommerfeld quantization rule (Bender and Orszag $^{8}$ )

$$
\frac{1}{\epsilon} \int_{z_{1}}^{z_{2}}[-Q(z)]^{\frac{1}{2}} \mathrm{~d} z=2 \pi(n+1 / 2)
$$


Bertin, Millet and Bouche, J. of the Acoustical Society of America, p. 21

where $z_{1}$ and $z_{2}>z_{1}$ are first-order turning points and $\operatorname{Re}[Q(z)]<0$ for $z_{1}<z<z_{2}$. For the general case in which $\Omega$ is a complex frequency, we note that $z_{1}$ and $z_{2}$ are not zeros of the complex function $Q$ and thus, equation (17) no longer holds. New conditions can be derived from matching conditions of the approximations to $p(z)$. These matching conditions translate into constraints on both the real and imaginary parts of $Q$. The details of the algebra are given in the appendix for an arbitrary profile $c(z)$. For the sake of simplicity, we restrict the discussion here to the profile displayed in figure 5 . Denoting $z_{1}$ and $z_{2}$ two consecutive zeros of $\operatorname{Re}(Q)$, the constraint on the real part of $Q$ is given by equation (17) in which $Q$ must be replaced by $\operatorname{Re}(Q)$. The constraint on the imaginary part of $Q$ is

$$
\frac{1}{\epsilon} \int_{z_{1}}^{z_{2}} \operatorname{Im}[-Q(z)]^{1 / 2} \mathrm{~d} z=\frac{2}{3 \epsilon} \operatorname{Re}\left[\frac{Q^{3 / 2}\left(z_{2}\right)}{Q^{\prime}\left(z_{2}\right)}-\frac{Q^{3 / 2}\left(z_{1}\right)}{Q^{\prime}\left(z_{1}\right)}\right]+O(\epsilon)
$$

which reduces to

$$
\frac{1}{\epsilon} \int_{0}^{z_{0}} \operatorname{Im}(-Q(z))^{1 / 2} \mathrm{~d} z=\frac{2}{3 \epsilon} \operatorname{Re}\left[\frac{Q^{3 / 2}\left(z_{0}\right)}{Q^{\prime}\left(z_{0}\right)}\right]+O(\epsilon)
$$

when the oscillating region extends to the ground; i.e. $\operatorname{Re}[Q(z)]<0$ for $0 \leq z<z_{0}$. Through algebraic manipulations, we can generalize the condition (18) to an arbitrary number of zeros of $\operatorname{Re}(Q)$. It is worth mentioning that equation (18) is based on the fact that each zero is simple and thus, this approach fails for altitudes $z$ at which two oscillating regions merge. This is a familiar restriction which excludes a small neighborhood of cusp regions in the complex $K$-plane. 
Bertin, Millet and Bouche, J. of the Acoustical Society of America, p. 22

Following the above reasoning, the real turning points $z(K)$ and the oscillating regions are first obtained by solving $Q(z ; K, \Omega)=0$, for a fixed frequency $\Omega$. As the mapping of a given $K$ into the complex $z$-plane is generally multivalued, we denote the various images of $K$ that lie along the real axis by $z_{m}(K)$. The mapping of a contour in the complex $K$-plane into the $z$-axis may be rendered single-valued by constructing a multisheeted $K$-plane with $n$ sheets, each corresponding to a single oscillating region $z_{m}<z<z_{m+1}$. In some sense, the profile $c(z)$ can be seen as a potential, each of its oscillating regions being associated with one curve $\gamma_{n}$ (lying on a single sheet) in the complex $K$-plane on which eigenvalues are distributed according to the real part of the Bohr-Sommerfeld condition (17). The appearance of a cusp in the spectrum structure (which is a branch point of the square root in (17)) at a particular phase velocity serves as a warning signal that the partition into two disconnected sheets is not possible. Such a cusp corresponds to a local maximum (a double turning point) of $c(z)$ at which two oscillating regions merge and, thus, two sheets are connected in the complex plane, as illustrated in figure 5 .

Equation (18), while hardly trivial, is readily solved (for a fixed $\Omega$ ) by standard methods for complex root determination. Here we use a Newton-Raphson method for finding $K$, subject to the constraint $z_{m}<z<z_{m+1}$. Figure 5 shows that typical curves $\gamma_{n}(n=1,2,3)$ merge at $k_{r}=\omega_{r} / c$, where $c=320 \mathrm{~m} \cdot \mathrm{s}^{-1}$. Similar results are shown in figures 6 and 7 for both the ERA-Interim and the 91-level profiles. As can be seen from figures 6 and 7 , 
Bertin, Millet and Bouche, J. of the Acoustical Society of America, p. 23

the most sensitive modes issuing from the cusp at $c_{r} \simeq 335 \mathrm{~m} \cdot \mathrm{s}^{-1}$ converge to the path in which the imaginary part $k_{i}$ (orange curve) is minimal, so that, in view of the results in section III, the eigenvalue computation can be restricted to the invariant subspace associated with the neighborhood of this curve, which will be called $\gamma_{1}$. Specifically, an implicitly restarted Krylov-Arnoldi algorithm $\left(\right.$ Watkins $\left.^{38}\right)$ is used with a shift-and-invert method, or a Cayley transform $\left(\right.$ Lehoucq ${ }^{26}$, Meerbergen et al. ${ }^{29}$ ), centered in the middle of $\gamma_{1}$. Each restart gives a better approximation to the desired invariant subspace. Repeated restarts lead to convergence. Since Krylov subspace methods have good convergence properties when combined with Tchebychev collocation points $\left(\right.$ Beattie $\left.^{6}\right)$, we find a good agreement between both the QR algorithm (LAPACK Fortran library) and the Arnoldi algorithm (ARPACK Fortran library); the relative error of the eigenvalues being below $10^{-6}$. Figure 8 shows results for the profile $c_{1 a}(z)$. In terms of computational cost, there is a huge benefit in doing so, especially when the frequency is high (figure $8 \mathrm{~b}$ ). While the $\mathrm{QR}$ algorithm needs more than $7 \mathrm{~h}$ to compute a signal up to $1 \mathrm{~Hz}$ (with 512 frequency samples) the application of the Arnoldi algorithm in the vicinity of $\gamma_{1}$ leads to a total computation time as short as several minutes $(2 \%)$ on a single processor.

It should be stressed that the main difficulties in applying the Krylov-Arnoldi algorithm are to determine (1) the dimension $m$ that warrants the accuracy of the required eigenvalues and (2) the number $n$ of desired eigenvalues $(n<m)$. Given a vector $x$ and a matrix 
Bertin, Millet and Bouche, J. of the Acoustical Society of America, p. 24

$A$, the Krylov space of $A$ is the $m$-dimensional subspace spanned by $x, A x, \ldots, A^{m-1} x$. The basic idea in Krylov subspace or projection methods is to construct eigenpairs (the Ritz pairs) in the Krylov subspace $\mathcal{K}_{m}$ so that the component orthogonal to that space is sufficiently small for the Ritz pairs to be good approximations to eigenpairs of $A$. A convenient way to do this is given by the Arnoldi factorization (Watkins ${ }^{38}$ ) which provides the eigenpairs of $A$ from the eigenpairs of smaller matrices. Such a method, however, is entirely dependent on the choice of the starting vector $x$. Here, we require $x$ to be rich in the subspace spanned by the eigenvectors corresponding to eigenvalues that are nearest the curve $\gamma_{1}$ with very small components in the direction of the other eigenvectors. We follow the implicitly restarted Arnoldi method (Watkins ${ }^{38}$ ) and adaptively refine $x$ to be a linear combination of the $n$ eigenvalues without explicitly computing a new Arnoldi factorization.

The larger $m$ is, the better is our chance that the space $\mathcal{K}_{m}$ contains good approximations to desired eigenvectors. However, the convergence of the implicitly restarted Arnoldi method is not uniform $\left(\right.$ Lehoucq $^{27}$ ) and there is no known a priori value of $m$ leading to optimal convergence. Even though convergence techniques can considerably decrease the numerical cost of the Krylov approach, in the present study, however, the dimension of the Krylov space is fixed to $m=0.01 N$, where $N$ is the matrix size (typically $m=20$ ). The number $n$ of eigenvalues plays an essential role since the conditioning of the $m-n$ unwanted eigenvalues strongly affects the convergence of the implicitly restarted algorithm (Beattie ${ }^{7}$.) In this 
Bertin, Millet and Bouche, J. of the Acoustical Society of America, p. 25

study, we used a QR computation at the frequency of $1 \mathrm{~Hz}$ to estimate the number of eigenvalues lying on the curve $\gamma_{1}$ as $\omega_{r} \rightarrow 2 \pi$. Based on the numerical results, we have 3 to 5 eigenvalues, depending on the profile (figure 11a). Then, for each frequency $\omega$, the Arnoldi algorithm was applied to find the $n=10$ closest eigenvalues to $\gamma_{1}$.

Recall that for the range-dependent approach, the sensitivity of eigenvalues strongly depends on the local vertical profile $c(z ; R)$ so that, modes may become dominant over a fairly narrow range of distances or new modes (issuing from a single or several new paths) may litterally appear/disappear. The higher the horizontal variation, the larger the probability is to have a significant contribution from these modes to the ground-based signal. That is the main reason for which the sound speed profile evolution $c(z ; R)$ has to be studied before proceeding with our reduction method. Once the relevant curves $\gamma_{n}(\omega, R)$ (and plausible cusps) have been computed over the frequency-range domain, a Krylov-Arnoldi algorithm can be used to compute the invariant subspace associated with the most sensitive eigenvalues.

The eigenvalues can finally be analyzed as $R$ varies with a Newton-Raphson algorithm or a step-by-step Krylov-Arnoldi algorithm.

\section{B. Reduced models for range-dependent media}

The above reduction technique is based on the eigenvalue sensitivities which means that the reduced model preserves the overall structure under sufficiently small perturbations of the vertical profile. The duality between range-dependence and perturbation (revealed 
Bertin, Millet and Bouche, J. of the Acoustical Society of America, p. 26

by equation (1)) can be used to extend the validity domain of the reduced model to rangedependent media. From a numerical standpoint, this task reduces in following the selected eigenvalues as the vertical profile slowly varies along the source-receiver path.

Recall that the spatial eigenvalues $k_{j}(\omega)$ have been introduced as solutions of the dispersion relation when the contour $F$ in the complex $\omega$-plane differs from the real axis. Although a straightforward interpretation is missing, the temporal branches $k_{j}(\omega)$ are objects that are naturally involved whenever the initial contour is gradually displaced upward from the real axis. According to the frequency shifting property of the Fourier-Laplace transform, the ground-based signal can be obtained from the classical FFT algorithm (for $\omega=\omega_{r}$ ) together with the mapping $p(t) \mapsto p(t) e^{\omega_{i} t}$. A trapezoidal rule was used to compute the phase in (7), using 12 intermediate ranges $R$ between the source and the station. While the complete computation involves 580 modes, the implicitly restarted Krylov-Arnoldi method is used to find the 3 most sensitive modes that correspond to the tropospheric waveguide, with a cost of $2 \%$ of the total run time, as previously indicated (figure 8). Here, we use a simplified source model defined by

$$
s(t)=\left[1-\cos \frac{\pi t}{2}\right] \sin \left(\pi t+\frac{\pi}{2}\right)
$$

together with $s(t)=0$ for $t<0 \mathrm{~s}$ and $t>4 \mathrm{~s}$. Hence, the power spectra at the station I30JP $(R=243 \mathrm{~km})$ is simply the product of the Fourier Transform of (20) and (7).

It is important to emphasize that the tropospheric sheet in the complex $k$-plane (fig- 
Bertin, Millet and Bouche, J. of the Acoustical Society of America, p. 27

445

where $J$ is the subset of most sensitive modes and

$$
p_{j}\left(\omega_{r}, r\right)=\frac{\phi_{j}^{2}(0 ; \omega, R)}{\sqrt{k_{j}(\omega, R)}}\left|\hat{s}\left(\omega_{r}\right)\right|,
$$
Fourier transform. the source term (20). Then, we take its Fourier transform so as to obtain

ure 5) contains all the necessary information for waveform synthesis. This sheet is an attracting sector for the mode trajectories (as the frequency increases) that are most sensitive to low altitude perturbations thereby leading to a physical interpretation in terms of atmospheric structures. Futhermore, the modes emerge from a cusp at a specific cutoff frequency $\omega_{0}\left(0.09 \mathrm{~s}^{-1}, 0.39 \mathrm{~s}^{-1}\right.$ and $0.71 \mathrm{~s}^{-1}$ for the first, second and third mode, respectively). On the other hand, since $\omega_{i}>0$ these modes lie in the upper half of the complex $k$-plane and thus, the contribution of the various poles vanishes as $\omega_{r} \rightarrow+\infty$, as shown in figure 4 . We can therefore take advantage of these results to obtain an approximation of the temporal

To develop the leading-order term of the inverse Fourier transform, we first rewrite (7) along an arbitrary fixed spatio-temporal ray $r /(t-\tau)=v$, where $\tau \omega$ is the phase shift of

$$
p(r, 0 ; r / v)=\frac{i \mathrm{e}^{-i \frac{\pi}{4}}}{4 \pi \sqrt{2 \pi r}} \sum_{j \in J} \mathrm{e}^{\omega_{i}\left(\frac{r}{v}+\tau\right)} \int_{-\infty}^{+\infty} p_{j}\left(\omega_{r}, r\right) \exp \left\{\frac{i}{\epsilon} \Phi_{j}\left(\omega_{r}, R\right)\right\} \mathrm{d} \omega_{r}
$$

where $\hat{s}$ is the Fourier transform of (20). In the limit $\epsilon \rightarrow 0$ with $R$ fixed, the integral may be evaluated asymptotically by the well-known stationary phase method. The phase function 
Bertin, Millet and Bouche, J. of the Acoustical Society of America, p. 28

460

of $(21)$ is

$$
\Phi_{j}\left(\omega_{r}\right)=\int_{0}^{R} k_{j}\left(s, \omega_{r}\right) \mathrm{d} s-\frac{\omega_{r} R}{v} .
$$

461

The points of stationary phase are given by the roots of $\Phi_{j}^{\prime}\left(\omega_{r}\right)=0$ or, from $(23)$,

$$
\int_{0}^{R} \frac{\partial k_{j}}{\partial \omega} \mathrm{d} s-\frac{R}{v}=0
$$

462

subject to the constraint $\omega_{0}<\omega<2 \pi$, where $\omega_{0}$ is the cut-off frequency $\left(\omega_{0}=0.09 \mathrm{~s}^{-1}\right.$ for the first most sensitive mode).

Two distinct behaviors are possible. Whenever $v_{1}<v<v_{2}$, (24) has a unique real solution that we denote by $\omega_{r}=\bar{\omega}(R, v)$, as shown in figure 9 . Thus, among all the frequencies contained in the source, the atmosphere filters out, along each ray $x / t=v$ one particular frequency $\bar{\omega}$ given by (24). In the opposite case, where the velocity $v$ is greater than $v_{2}$ or less than $v_{1}$, there is no solution to (24) and thus, the integral vanishes (Bender and Orszag $^{8}$ ). Indeed, once the velocity front $v_{1}$ has reached the infrasound station, the atmosphere returns to the rest state.

The leading-order contribution arising from the first dominant mode $(j=1)$ in expression (21) can be evaluated according to the general formulas given in Bender and Orszag ${ }^{8}$. As $\epsilon \rightarrow 0$, the impulse response associated with this mode reduces to

$$
p(r, v) \sim \frac{i{\overline{\phi_{1}}}^{2}(R, v) \mathrm{e}^{i \frac{\overline{\Phi_{1}(R, v)}}{\epsilon}+\omega_{i}\left(\frac{r}{v}+\tau\right)}}{4 \pi \sqrt{r}\left[\frac{\left.\overline{k_{1}}(R, v)\right)}{\epsilon} \int_{0}^{R} \frac{\partial^{2} k_{1}}{\partial \omega^{2}}(s, v) \mathrm{d} s\right]^{\frac{1}{2}}}\left[1+\frac{\alpha(R, v)}{\epsilon}+\ldots\right]
$$


474

where

$$
\alpha=\frac{5 i p_{1}\left[\Phi_{1}^{(3)}\right]^{2}+12 i p_{1}^{(2)} \Phi_{1}^{(2)^{2}}-12 i p_{1}^{(1)} \Phi_{1}^{(3)} \Phi_{1}^{(2)}-3 i p_{1} \Phi_{1}^{(4)} \Phi_{1}^{(2)}}{12 p_{1}\left[\Phi_{1}^{(2)}\right]^{3}}
$$

evaluated at $\bar{\omega}$, with the notation

$$
\Phi_{1}^{(l)}=\frac{\partial^{(l)} \Phi_{1}}{\partial \omega^{(l)}}(R, \omega), \quad p_{1}^{(l)}=\frac{\partial^{(l)} p_{1}}{\partial \omega^{(l)}}(R, \omega) \quad \text { and } \quad \phi_{1}=\phi_{1}(0 ; R, \omega) .
$$

The response takes the form of a wavepacket in the $(r, t)$ plane, as shown in figure 9 . The wave packet is confined within a wedge bounded by the two rays $r / t=328 \mathrm{~m} \cdot \mathrm{s}^{-1}$ and $r / t=335 \mathrm{~m} \cdot \mathrm{s}^{-1}$. Inside the packet, the contribution is dominated by the stationary phase. For $\alpha=0$, the wave packet (25) is compared to the FFT with 580 modes in figure 10 . We find a very good overall approximation of the waveform with a single mode (the first most sensitive mode issuing from the cusp at $c_{r} \simeq 335 \mathrm{~m} \cdot \mathrm{s}^{-1}$ ) evaluated at the frequency $\bar{\omega}$. In practice, we apply the Krylov-Arnoldi algorithm to find the eigenvalues that are nearest the curve $\gamma_{1}$ in the complex $k$-plane. Once the eigenvalues $k_{j}$ are obtained for a fixed frequency, a Newton-Raphson method is used to solve $\Phi_{1}^{\prime}=0$ for a given $v$, without significant supplementary CPU time. In striking contrast to other numerical methods, the complex waveform can be computed from a single mode evaluated at the frequency $\bar{\omega}$, with a CPU time of typically 1-10 seconds.

The question of how relevant the stationary phase approximation is to practical applications is intimately related to confidence intervals. Through algebraic manipulations, we can 
Bertin, Millet and Bouche, J. of the Acoustical Society of America, p. 30

find a higher-order asymptotic expansion to (21). However, the expansion requires higherorder derivatives $k_{j}^{(3)}, k_{j}^{(4)}, \ldots$ (see $\left.(26)\right)$ and thus, accurate means of solving the dispersion relation in the complex $k$-plane. Such an approach would require higher computer time. It led us to believe that our approach, which gives an approximate waveform at a considerably lower CPU time, promises to be more fruitful than seeking the maximum precision for a fixed atmospheric state. In other words, we hypothesize that, the higher the sampling size, the better the obtained statistics, which is the ultimate goal of many infrasound studies. In the meantime, the present paper has shown how the CPU time can be considerably reduced, through the analytic continuation of the range-dependent normal mode method in the complex $k$-plane, which provides relevant statistics for applications.

\section{On the role of atmospheric uncertainties}

According to the discussion of section IV, the propagation mechanisms that occur just after the Fukushima explosion are dominated by three modes that evolve on a slow length scale $\epsilon^{-1}$, where $\epsilon$ is defined by equation (1). These modes trigger a tropospheric arrival which is in good qualitative agreement with the first arrival of the recorded signal (see figure 1). However, the direct computation predicts also a stratospheric 'tail' which is several orders of magnitude weaker than the tropospheric arrival (blue signal, figure 12). This is clearly not what was recorded at the Japanese station.

An interesting aspect of using a complex frequency is that the imaginary parts of eigen- 
Bertin, Millet and Bouche, J. of the Acoustical Society of America, p. 31

values may be arranged so that the tropospherically-ducted modes lie along the path $\gamma_{1}$ in the complex $k$-plane as $\omega_{r} \rightarrow \infty$. Since this path can be obtained at very low numerical cost through a WKB analysis, the propagation problem can be projected onto an invariant subspace corresponding to these modes. Hence, we obtain a reduced model for the tropospheric arrival by seeking eigenvalues that are close to $\gamma_{1}$ with a Krylov-Arnoldi algorithm. One value of the reduced model lies in the reduction of the order (the number of modes) which is based on the sensitivites of the eigenvalues over a given frequency range. By rearranging the modes by decreasing sensitivities (and decreasing contribution to the ground-based signal), the reduced model preserves the overall structure of waveforms under perturbations of the troposphere. In a sense, the requirement for the reduced model to be reasonably robust to variations of the profile $c(z)$ is similar to the so-called structural stability property in mathematics.

Figure 11e-g shows the ground based signals at I30JP obtained from three synthetic atmospheric conditions, with an effective sound speed at the local tropospheric maximum significantly smaller, equal or significantly larger than that at ground level. These profiles are obtained from $c_{1 a}$ pertubed by a Gaussian envelope centered at the altitude of maximum effective sound speed, as shown in figure 11a. The relevant eigenvalues (close to $\gamma_{1}$ ) are computed with a Krylov-Arnoldi algorithm. We obtain 2, 3 or 4 eigenvalues at $1 \mathrm{~Hz}$ (figure 11b-d), depending on the profile $c(z)$. The associated ground-based signals obtained 
Bertin, Millet and Bouche, J. of the Acoustical Society of America, p. 32

with the reduced model (truncated to 2, 3 and 4 modes) are in excellent agreement with that obtained with the full model (i.e. with 580 modes). Being able to represent the signals with a small number of modes offers an argument that the reduced model may be used to derive statistical results for a wide range of tropospheric conditions.

In the above reduced model, it was explicitly assumed that the vertical sound speed profiles are fixed and obtained from the European Centre for Medium-Range Weather Forecasts (ECMWF). This assumption, although providing a good approximation of the tropospheric wavepacket, cannot be used to compute the stratospheric arrival. Indeed, it is now generally recognized that small-scale gravity waves are filtered out of the ECMWF fields. At leading order, the amplitude of the upward propagating waves grows in altitude as $1 / \sqrt{\rho}$, where $\rho$ is the density of the atmosphere. Above $60 \mathrm{~km}$, the amplitude of the unresolved waves can reach $10 \%$ of the sound speed (Drob et al. ${ }^{17}$ ). Even though in the lower atmosphere the amplitude of these waves represents a small fraction of the overall variations of the average background state, a quantitative theory of waveform changes due to gravity waves is not available.

Figure 12 provides further calculations, using as the input profile a slightly perturbed version of $c_{1 a}$ (figure 1). For simplicity, the perturbation is modelled by a single upward gravity wave modulated by a Gaussian envelope that mimics the gravity wave breaking. The main effect of the localized perturbation is to slightly redistribute the sensitivities so 
Bertin, Millet and Bouche, J. of the Acoustical Society of America, p. 33

that a second subset of sensitive eigenvalues arises from another cusp region, as shown in figure 12b. The associated modes trigger a stratospheric arrival (green signal) that appears to be dominant for specific vertical wavelengths, even though the perturbed sound speed remains smaller than the value at ground level. The effect is, however, non-uniform being largest for a vertical wavelength of about $1 \mathrm{~km}$.

Following the method described in section IV, the two arrivals can be obtained by applying the Krylov-Arnoldi algorithm (figure 12c) twice and adding the new eigenvalues to the reduced model. While the perturbation amplitude is limited to less than $1 \%$ of the effective sound speed, it is evident from figures 12 and 1 that there is a considerably improved overall agreement between calculated and recorded signals. Finally, note that the main difficulty is to determine which phase dominates in the computed signal. A priori this requires the detailed analysis of the subsets containing the most sensitive eigenvalues in the complex $k$-plane.

\section{Conclusion}

We have examined the pressure field generated by a point source explosion within a background atmospheric state given by a combination of ECMWF data and empirical reference models. The impact on long-range infrasound propagation of small departures from a given profile was studied through perturbation theory. In practice, these imperfections may stem from atmospheric structures that are not considered (e.g. gravity waves) or they 
Bertin, Millet and Bouche, J. of the Acoustical Society of America, p. 34

may simply be the result of horizontal variation. While a consensus seems to have emerged that gravity waves with length scales of less than about $100 \mathrm{~km}$ are filtered out in available atmospheric specifications, it is not clear whether classical high frequency techniques are able to capture the effects of these scales.

In this paper, we used a range-dependent normal mode approach with no constraint on the frequency. The analysis of the vertical structure of the wave equation in the complex wavenumber plane (the so-called $k$-plane) indicates that only a few eigenvalues are involved in long-range propagation of infrasounds. These modes, which are sensitive to key atmospheric features, can be computed with a high degree of accuracy through a Krylov subspace process that uses a shift-and-invert strategy. Sensitivity arguments indicate that these modes are close to curves in the complex $k$-plane that may be obtained through the analytic continuation of the classical WKB analysis. The process time of our reduced model is two orders of magnitude less than the computation of the complete spectrum with a QR algorithm. It therefore appears that a low order reduced model based on a few suitable eigenvalues can provide the impulse response over large ranges of frequencies. This reduced model maintains full generality and can be applied to range-dependent media so that waveforms can be recovered by using a stationary phase method. In this case the process time is no longer than several seconds on a single processor.

Our reduced model gives insights into the role of atmospheric structures in the wave- 
Bertin, Millet and Bouche, J. of the Acoustical Society of America, p. 35

584

585

586

587

588

form features. By means of a wavelet-based decomposition of the profile perturbation, we have shown that the components leading to the most sensitive eigenvalues give regions and wavelengths of atmospheric structures involved in the propagation. Other regions of the background atmosphere are not relevant to the computed waveform. Indeed, our reduced model allows sensitivity analysis and statistical studies at minimum CPU time. 
Bertin, Millet and Bouche, J. of the Acoustical Society of America, p. 36

\section{WKB approximation}

We consider here the wave equation

$$
\epsilon^{2} \frac{d^{2} p}{d z^{2}}=Q(z ; K, \Omega) p
$$

where $\epsilon$ is a small parameter, with

$$
Q(z ; K, \Omega)=K^{2}-\Omega^{2} / c^{2}(z),
$$

where $K$ and $\Omega$ are complex. Furthermore, we make the assumption that $\operatorname{Im}[Q] \ll \operatorname{Re}[Q]$ so that (27) refers to the complex perturbation of the real problem fully treated by Bender and Orszag $^{8}$. By limiting the asymptotic expansion of the phase to order 1, the classical approach leads us to express the solution as a linear combination of two independant solutions:

$$
p(z)=Q^{-1 / 4}(z)\left[A e^{\frac{1}{\epsilon} \phi(z)}+B e^{-\frac{1}{\epsilon} \phi(z)}\right]
$$

where $\phi^{\prime}=Q^{1 / 2}$ and $A$ and $B$ are scalar constants depending on boundary conditions. Since $Q(z)$ is close to the real axis, the solution clearly has two distinct behaviors along the $z$-axis: the solution is dominated by a real exponential for $\operatorname{Re}[Q(z)]>0$ and is mostly oscillating in regions where $\operatorname{Re}[Q(z)]<0$.

In the vicinity of points $z^{*}$ such that $\operatorname{Re}\left[Q\left(z^{*}\right)\right]=0$, the asympotic expansion is no longer valid. Nevertheless, a first order Taylor series expansion can be obtained through a change of variable $z \rightarrow Z(z ; \epsilon)$, thereby leading to the Airy equation $\mathrm{d}^{2} / \mathrm{d} Z^{2} p=Z p$. 
Bertin, Millet and Bouche, J. of the Acoustical Society of America, p. 37

602 603 604

The solution in this critical layer can be expressed as a linear combination of Airy functions. Matching arguments provide a direct linear relation between the coefficients of $(28),\left(A_{u}, B_{u}\right)$ and $\left(A_{l}, B_{l}\right)$ above and below the critical layer respectively;

$$
\left[\begin{array}{l}
A_{u} \\
B_{u}
\end{array}\right]=P\left[\begin{array}{l}
A_{l} \\
B_{l}
\end{array}\right] .
$$

Let the zeros of $\operatorname{Re}(Q)$ be sorted so that $0<z_{1}<z_{2}<\cdots<z_{N}$, and $P_{1}, P_{2}, \cdots, P_{N}$ be the corresponding passing matrices. From an iterative process, we can deduce from (29) the relation

$$
\left[\begin{array}{l}
A_{N} \\
B_{N}
\end{array}\right]=M\left[\begin{array}{l}
A_{1} \\
B_{1}
\end{array}\right] .
$$

where $M=P_{N} \cdots P_{2} P_{1}$.

Finally, we take into account the boundary conditions at $z \rightarrow \infty$ and $z=0$ to obtain conditions on $Q$. The boundary conditions can be written as linear relations involving the coefficients $\alpha_{1} A_{1}+\beta_{1} B_{1}=0$ for $z=0$, and $\alpha_{N} A_{N}+\beta_{N} B_{N}=0$ for $z \rightarrow \infty$. Adding these conditions to the system (30) provides a unique relation that is a necessary and sufficient condition for the existence of a solution for the problem (27)

$$
\beta_{1}\left(\alpha_{N} M_{11}+\beta_{N} M_{21}\right)=\alpha_{1}\left(\alpha_{N} M_{12}+\beta_{N} M_{22}\right)
$$

${ }_{614}$ Real and imaginary parts of the above relation lead to two expressions. First, we find 615 the Bohr-Sommerfeld quantization rule (17) where $z_{1}$ and $z_{2}$ correspond to ordered pairs 
Bertin, Millet and Bouche, J. of the Acoustical Society of America, p. 38

616

617

618

619

620

621 622

surrounding an oscillating area $(\operatorname{Re}[Q]<0)$. Then, since $\operatorname{Im}[Q] \neq 0$, there is also the necessary condition (18) or (19) in the case $\operatorname{Re}[Q]<0$ near the ground $\left(0<z<z_{1}\right)$. Note that (18) and (19) are given for homogeneous boundary conditions. In other words, the solution vanishes as $z \rightarrow \infty$ and there is a perfect reflection at $z=0\left(p_{1}^{\prime}(0)=0\right)$.

Acknowledgements This work was supported by the CEA and the European Commission's 7th Framework Programme under the project ARISE. The authors would like to thank C. P. Haynes for helpful comments. 
Bertin, Millet and Bouche, J. of the Acoustical Society of America, p. 39

\section{REFERENCES}

1. M. Abramowitz and I. Stegun, Handbook of Mathematical Functions (Dover Publications, New-York, 1964), 358-433.

2. E. Akervik, J. Hopffner, U. Ehrenstein and D. S. Henningson, Optimal growth, model reduction and control in a separated boundary-layer flow using global eigenmodes, $J$. Fluid Mech., 579, 305-314 (2007).

3. A. C. Antoulas, D. C. Sorensen and S. Gugercin, A survey of model reduction methods for large-scale systems, Contemp. Math., 280, 193-219 (2001).

4. Z. Bai, Krylov subspace techniques for reduced-order modeling of large-scale dynamical systems, Applied Numerical Mathematics, 43, 9-44 (2002).

5. A. Barbagallo, D. Sipp and P. J. Schmid, Input-output measures for model reduction and closed-loop control: application to global modes, J. Fluid Mech., 685, 23-53 (2011).

6. C. Beattie, M. Embree and J. Rossi, Convergence of restarted Krylov subspaces to invariant subspaces, SIAM J. Matrix Anal. Appl., 25, 1074-1109 (2001).

7. C. A. Beattie, M. Embree and D. C. Sorensen, Convergence of Polynomial Restart Krylov Methods for Eigenvalue Computations, SIAM Review 47(3), 492-515 (2005). 
Bertin, Millet and Bouche, J. of the Acoustical Society of America, p. 40

8. C. M. Bender and S. A. Orszag, Advanced Mathematical Methods for Scientists and Engineers I: asymptotic methods and perturbation theory, (Springer-Verlag, New-York, 1999), 504-533.

9. M. Bertin, C. Millet, D. Bouche and J.-C. Robinet, The role of atmospheric uncertainties on long range propagation of infrasounds, AIAA Paper, 2012-3346 (2012).

10. T. J. Bridges and P. J. Morris, Differential eigenvalue problems in which the parameter appears nonlinearly, J. of Computational Physics, 55(3), 437-460 (1984).

11. J. Candelier, S. Le Dizès and C. Millet, Shear instability in a stratified fluid when shear and stratification are not aligned, J. Fluid Mech., 685, 191-201 (2011).

12. J. Candelier, S. Le Dizès and C. Millet, Inviscid instability of a stably stratified compressible boundary layer on an inclined surface, J. Fluid Mech., 694, 524-539 (2012).

13. I. P. Chunchuzov, S. N. Kulichkov, O. E. Popov, R. Waxler and J. Assink, Infrasound scattering from atmospheric anisotropic inhomogeneities, Izv., Atmos. and Ocean. Phys., 47(5), 540-557 (2011).

14. I. Daubechies, Orthonormal bases of compactly supported wavelets, Comm. Pure Appl. Math., 41(7), 909-996 (1988).

15. J. Delville, L. Ukeiley, L. Cordier, J. P. Bonnet and M. Glauser, Examination of 
Bertin, Millet and Bouche, J. of the Acoustical Society of America, p. 41

large-scale structures in a turbulent plane mixing layer. Part 1. Proper orthogonal decomposition, J. Fluid Mech., 391, 91-122 (1999).

16. D. P. Drob, J. T. Emmert, G. Crowley, J. M. Picone, G. G. Shepherd, W. Skinner, P. Hays, R. J. Niciejewski, M. Larsen, C. Y. She, J. W. Meriwether, G. Hernandez, M. J. Jarvis, D. P. Sipler, C. A. Tepley, M. S. O’Brien, J. R. Bowman, Q. Wu, Y. Murayama, S. Kawamura, I. M. Reid and R. A. Vincent, An empirical model of the Earth's horizontal wind fields: HWM07, J. of Geophysical Research, 113, A12304, 1-18 (2008).

17. D. P. Drob, D. Broutman, M. A. Hedlin, N. W. Winslow and R. G. Gibson, A method for specifying atmospheric gravity wavefields for long-range infrasound propagation calculations, J. of Geophysical Research, 118(10), 3933-3943 (2013).

18. B. Galletti, C. H. Bruneau, L. Zannetti and A. Iollo, Low-order modelling of laminar flow regimes past a confined square cylinder, J. Fluid Mech., 503, 161-170 (2004).

19. A. Hay, J. T. Borggaard and D. Pelletier, Local improvements to reduced-order models using sensitivity analysis of the proper orthogonal decomposition. J. Fluid Mech. 629, 41-72 (2009).

20. A. E. Hedin, E. L. Fleming, A. H. Manson, F. J. Schmidlin, S. K. Avery, R. R. Clark, S. J. Franke, G. J. Fraser, T. Tsuda F. Vial and R. A. Vincent, Empirical wind model 
Bertin, Millet and Bouche, J. of the Acoustical Society of America, p. 42

for the upper, middle and lower atmosphere, Journal of Atmospheric and Terrestrial Physics, 58(13), 1421-1447 (1996).

21. M. A. H. Hedlin, C. D. de Groot-Hedlin and D. Drob, A study of infrasound propagation using dense seismic network recordings of surface explosions, Bulletin of the Seismological Society of America, 102, 1927-1937 (2012).

22. G. K. Batchelor, H. K. Moffatt and M. G. Worster, Perspectives in Fluid Dynamics: A collective introduction to Current Research, (Cambridge University Press, Cambridge, 2000), Chap. 4.

23. F. B. Jensen, W. A. Kuperman, M. B. Porter and H. Schmidt, Computational Ocean Acoustics (AIP Press, New-York, 1994), 257-322.

24. T. Kato, Perturbation Theory for Linear Operators (Springer-Verlag, Berlin Heidelberg, 1995), p. 619 .

25. S. N. Kulichkov, I. P. Chunchuzov and O. I. Popov, Simulating the influence of an atmospheric fine inhomogeneous structure on long-range propagation of pulsed acoustic signals, Izv., Atmos. and Ocean. Phys., 46(1), 60-68 (2010).

26. R. B. Lehoucq, Implicitly Restarted Arnoldi Methods and Subspace Iteration, SIAM J. Matrix Anal. and Appl., 23(2), 551-562 (2001). 
Bertin, Millet and Bouche, J. of the Acoustical Society of America, p. 43

27. R. B. Lehoucq, On The Convergence Of An Implicitly Restarted Arnoldi Method, SIAM J. Matrix Anal. Appl. 23, 551-562 (1999).

28. S. G. Mallat, Multiresolution representations and wavelets, Dissertations available from ProQuest, Paper AAI8824767 (1988).

29. K. Meerbergen and R. B. Morgan, Arnoldi Method with Inexact Cayley Transform in Z. Bai, J. Demmel, J. Dongarra, A. Ruhe and H. van der Vorst, Templates for the Solution of Algebraic Eigenvalue Problems: a Practical Guide, (SIAM, Philadelphia, 2000), 342343 (Section 11.2.3).

30. C. Millet, J. C. Robinet and C. Roblin, On using computational aeroacoustics for longrange propagation of infrasounds in realistic atmospheres, Geophysical Research Letters, 34(14), L14814 (2007).

31. G. R. North, Empirical orthogonal functions and normal modes, J. Atmos. Sci., 41(5), 879-887 (1984).

32. A. D. Pierce, Extension of the method of normal modes to sound propagation in an almost stratified medium, J. Acoust. Soc. Am., 37(1), 19-27 (1965).

33. J. W. Strutt (Baron Rayleigh), The theory of sound, Vol. 2, 2nd ed., revised and enlarged (MacMillan, London, New York, 1896), p. 132. 
Bertin, Millet and Bouche, J. of the Acoustical Society of America, p. 44

34. C. W. Rowlew, Model reduction for fluids using balanced proper orthogonal decomposition, Int. J. on Bifurcation and Chaos, 15(3), 997-1013 (2005).

35. B. Salimbahrami and B. Lohmann, Order reduction of large scale second-order systems using Krylov subspace methods, Linear Algebra and its Applications, 415(2-3), 385-405 (2006).

36. P. E. Srinivasan, D. Morrey, A. J. Bell, J. F. Durudola, E. B. Rudnyi and J. G. Korvink, Reduced order fully coupled structural acoustic analysis via implicit moment matching, Applied Mathematical Modelling, 33(11), 4097-4119 (2009).

37. L. N. Trefethen, Numerical Linear Algebra, (SIAM, Philadelphia, 1997), p. 258.

38. D. S. Watkins, The Matrix Eigenvalue Problem: GR and Krylov Subspace Methods, (SIAM, Philadelphia, 2008), 351-421.

39. R. Waxler, A vertical eigenfunction expansion for the propagation of sound in a downward-refracting atmosphere over a complex impedance plane, J. Acoust. Soc. Am., 112(6), 2540-2552 (2002).

40. R. Waxler, On the use of modal expansions to model broadband propagation in the nighttime boundary layer and other downward refracting atmospheres over lossy ground planes, J. Acoust. Soc. Am., 113(4), 2313 (2003). 
Bertin, Millet and Bouche, J. of the Acoustical Society of America, p. 45

724

725

726

41. R. Waxler, K. E. Gilbert and C. L. Talmadge, A theoretical treatment of the long range propagation of impulsive signals under strongly ducted nocturnal conditions, $J$. Acoust. Soc. Am., 124(5), 2742 (2008). 
Bertin, Millet and Bouche, J. of the Acoustical Society of America, p. 46

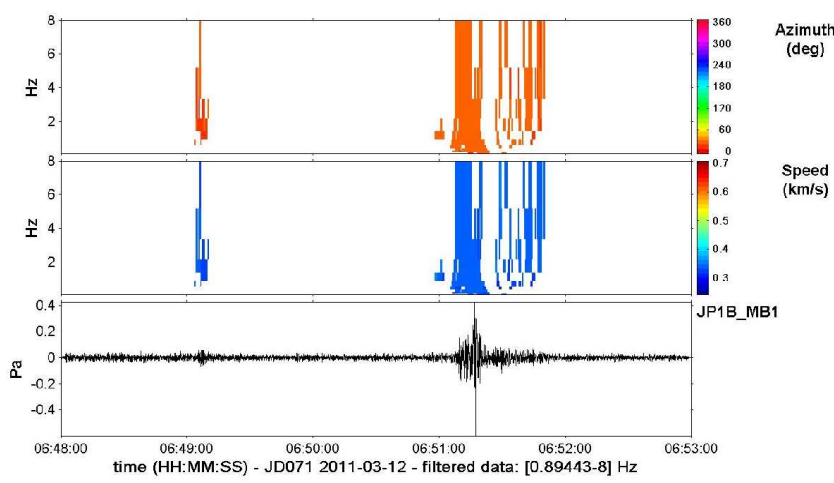

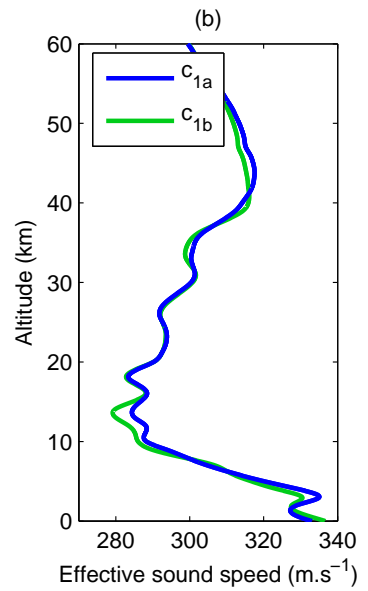

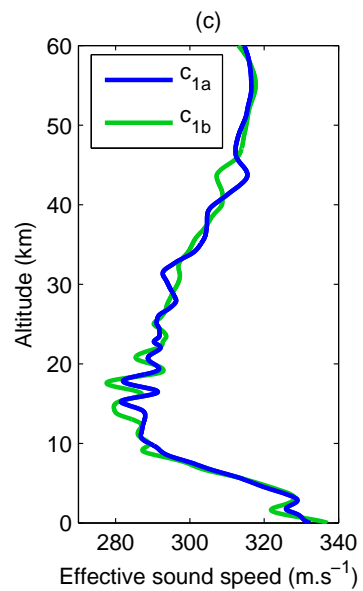

Figure 1: Data. (a): recorded signal at the Japanese infrasound station I30JP; effective sound speed profiles obtained from the ECMWF ERA-Interim dataset (b) and the ECMWF 91-level dataset (c) for locations that correspond to the Fukushima nuclear power plant $\left(c_{1 a}\right.$, $\left.c_{2 a}\right)$ and I30JP $\left(c_{1 b}, c_{2 b}\right)$. 
Bertin, Millet and Bouche, J. of the Acoustical Society of America, p. 47


Figure 2: The complex $k$-plane showing the trajectories of the local eigenvalues as $R$ varies for the ERA dataset (top) and the 91-level dataset (bottom). Blue: $r=0 \mathrm{~km}$; green: $r=243 \mathrm{~km}(\mathrm{I} 30 \mathrm{JP})$. 
Bertin, Millet and Bouche, J. of the Acoustical Society of America, p. 48

(a)

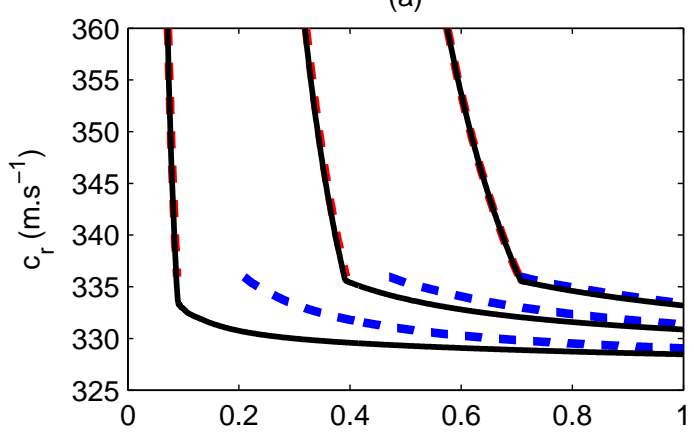

(b)

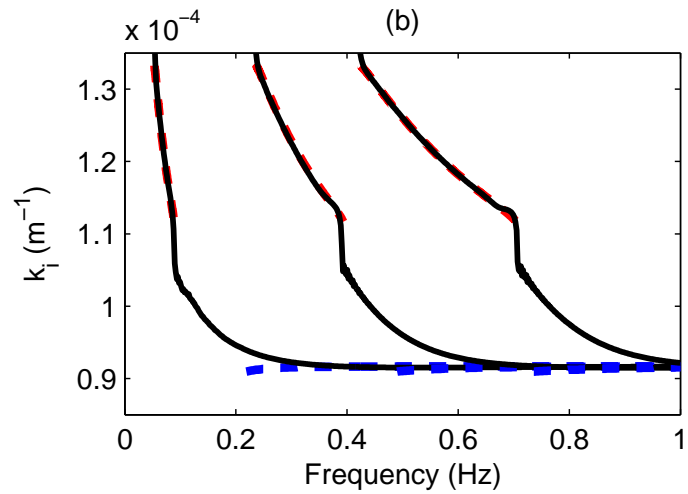

(c)



(d)

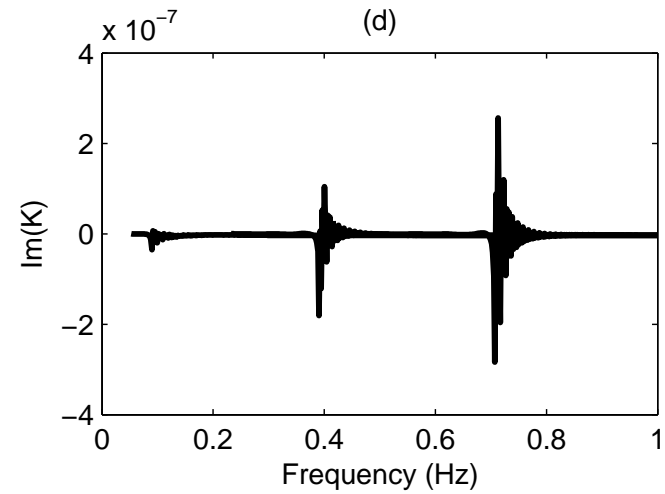

Figure 3: Phase velocity $\omega_{r} / k_{r}$ (a) and imaginary part $k_{i}$ (b) of the 3 most sensitive eigenvalues, as functions of the frequency $\omega_{r}$. Dashed lines represent the WKB approximations. Real (c) and imaginary (d) parts of sensitivities $K_{j m n}$ associated with the most sensitive eigenvalues for D10 wavelets $a=2, b=1, m=10$ and $n=1$ (see equation 9). Eigenvalues are computed for frequencies with a constant imaginary part of $\omega_{i}=0.03 . \mathrm{s}^{-1}$. 
Bertin, Millet and Bouche, J. of the Acoustical Society of America, p. 49
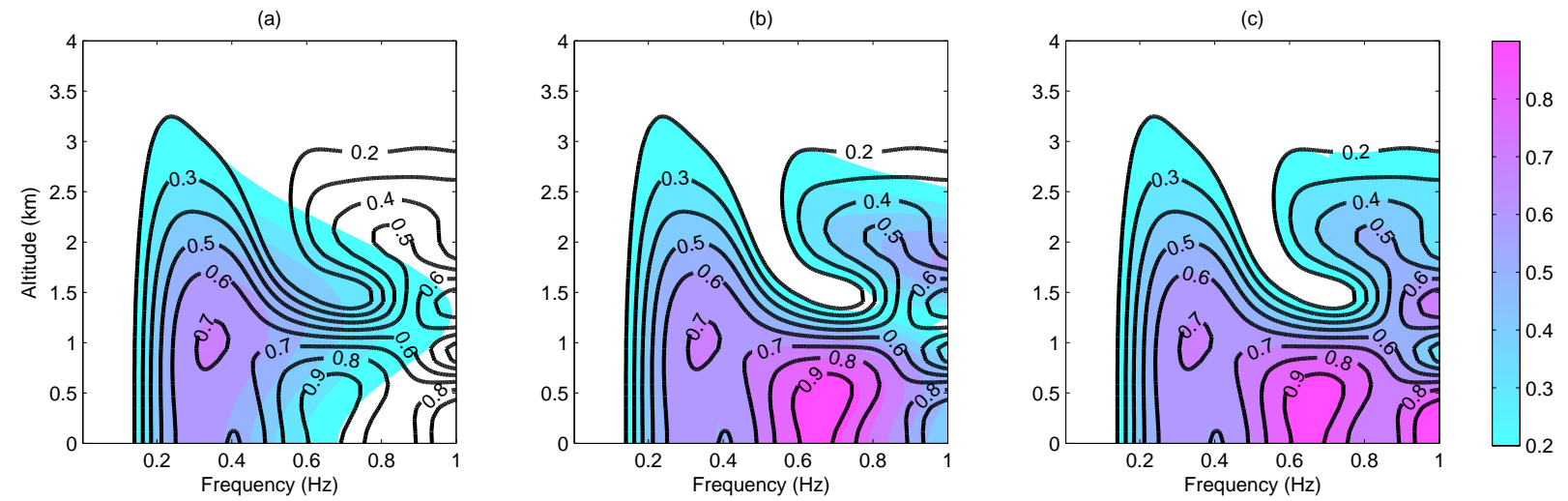

Figure 4: Pressure field contours in the $\omega_{r}-z$ plane at $r=243 \mathrm{~km}$, for the three most sensitive modes of figure 3. (a) first mode; (b) two modes and (c) three modes. Black levels give the pressure field $\left|\hat{p} / \hat{p}_{\max }\right|$ computed with 580 modes. Eigenvalues are computed for frequencies with an imaginary part $\omega_{i}=0.03 . \mathrm{s}^{-1}$. 
Bertin, Millet and Bouche, J. of the Acoustical Society of America, p. 50
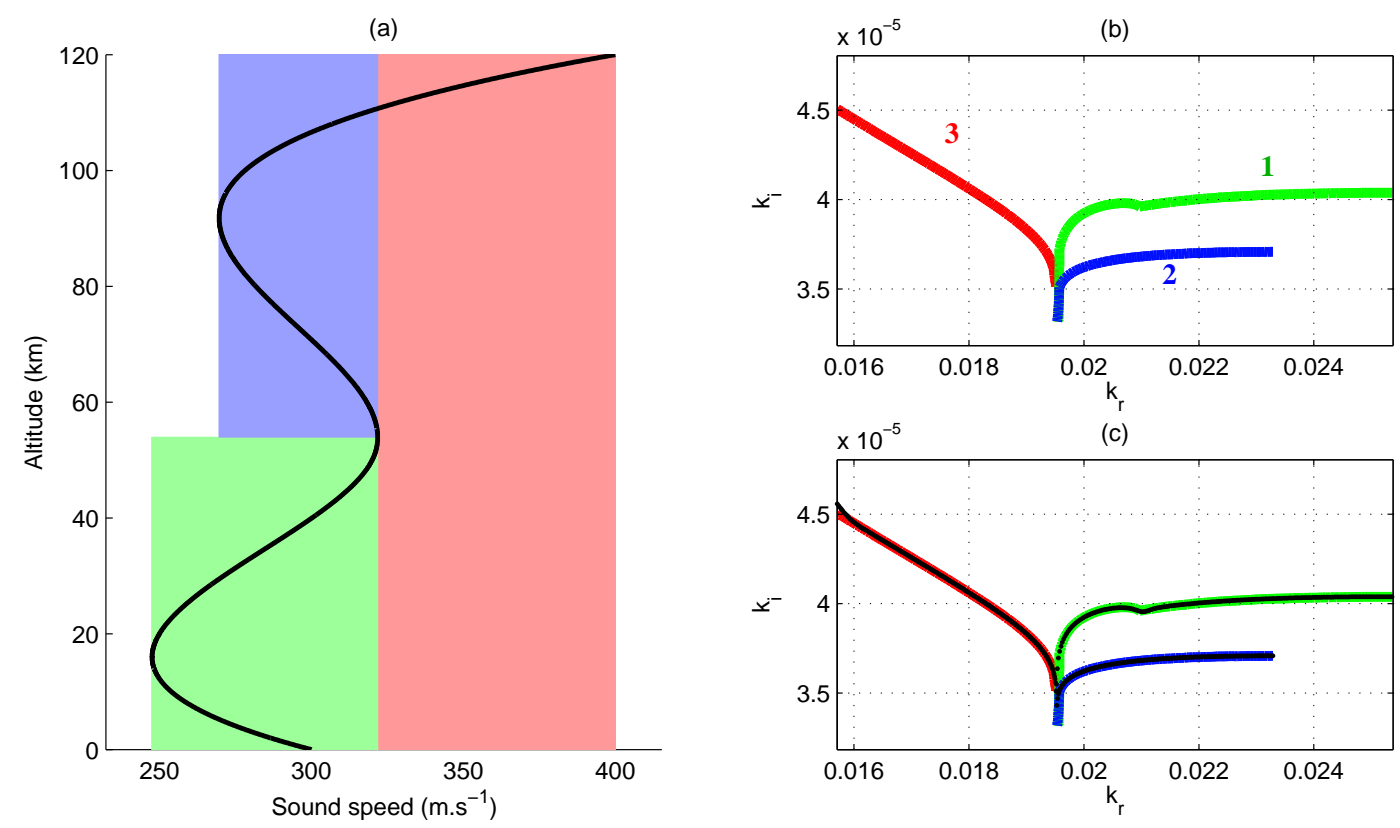

Figure 5: Typical effective sound speed profile (a) and various solutions of (17) (b) for $\omega=2 \pi+0.03 i$. The black circles (c) show the corresponding discrete spectrum (eigenvalues), as obtained with a $\mathrm{QR}$ algorithm. 
Bertin, Millet and Bouche, J. of the Acoustical Society of America, p. 51
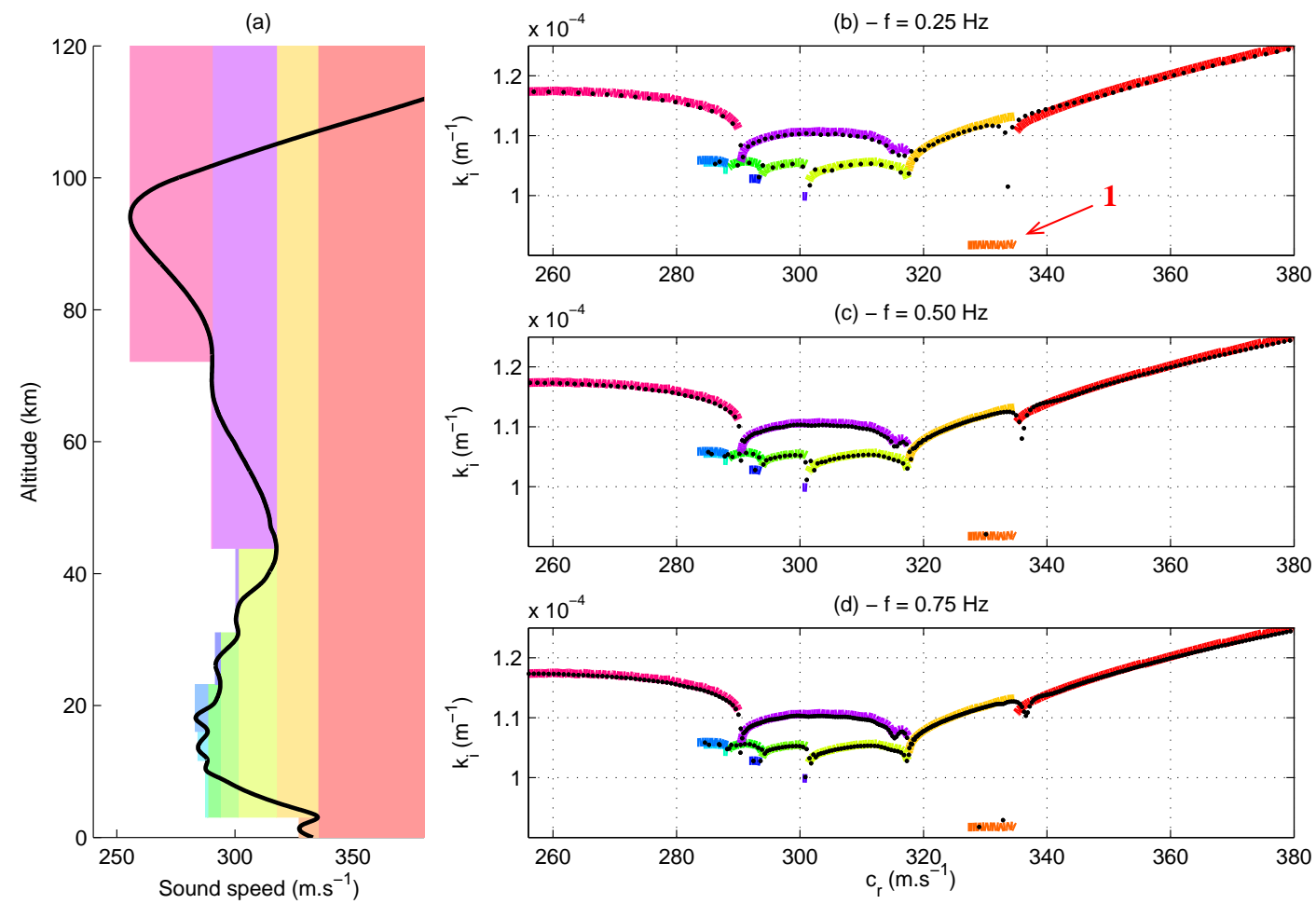

Figure 6: Various curves $\gamma_{n}$ in the complex $k$-plane for $\omega_{i}=0.03$ and the associated regions of the vertical profile $c(z)$. ERA-Interim data. 
Bertin, Millet and Bouche, J. of the Acoustical Society of America, p. 52
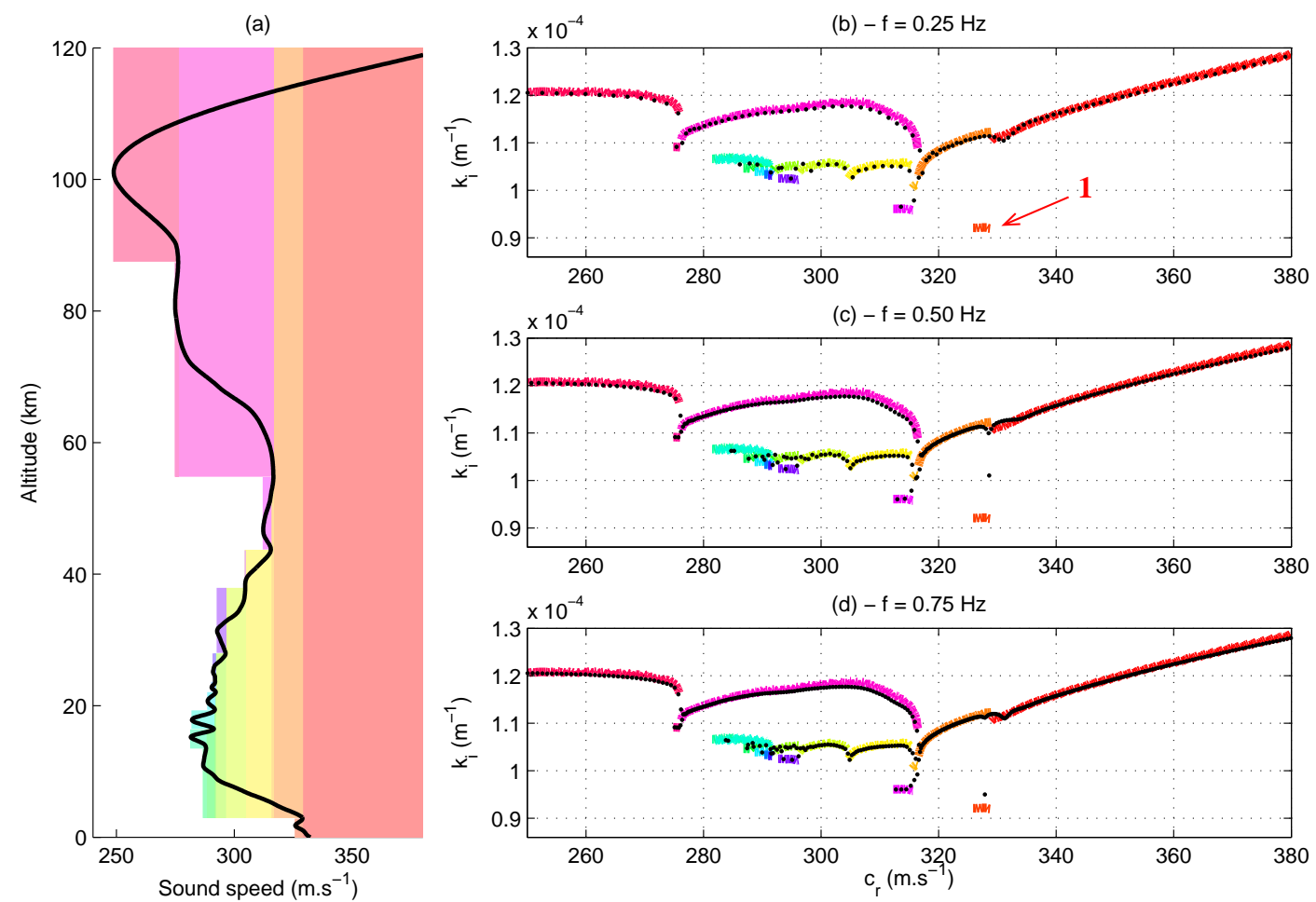

Figure 7: Various curves $\gamma_{n}$ in the complex $k$-plane for $\omega_{i}=0.03$ and the associated regions of the vertical profile $c(z)$. 91-levels data. 
Bertin, Millet and Bouche, J. of the Acoustical Society of America, p. 53

(a)

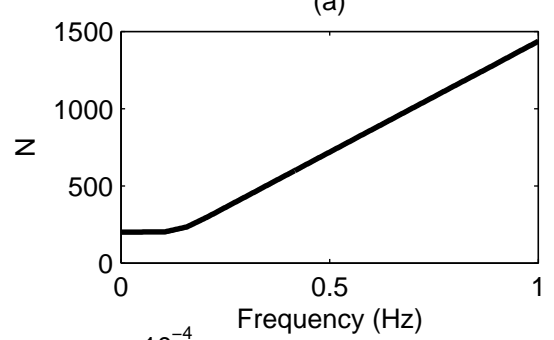

$\times 10^{-4} \quad$ Frequency $(\mathrm{Hz})$
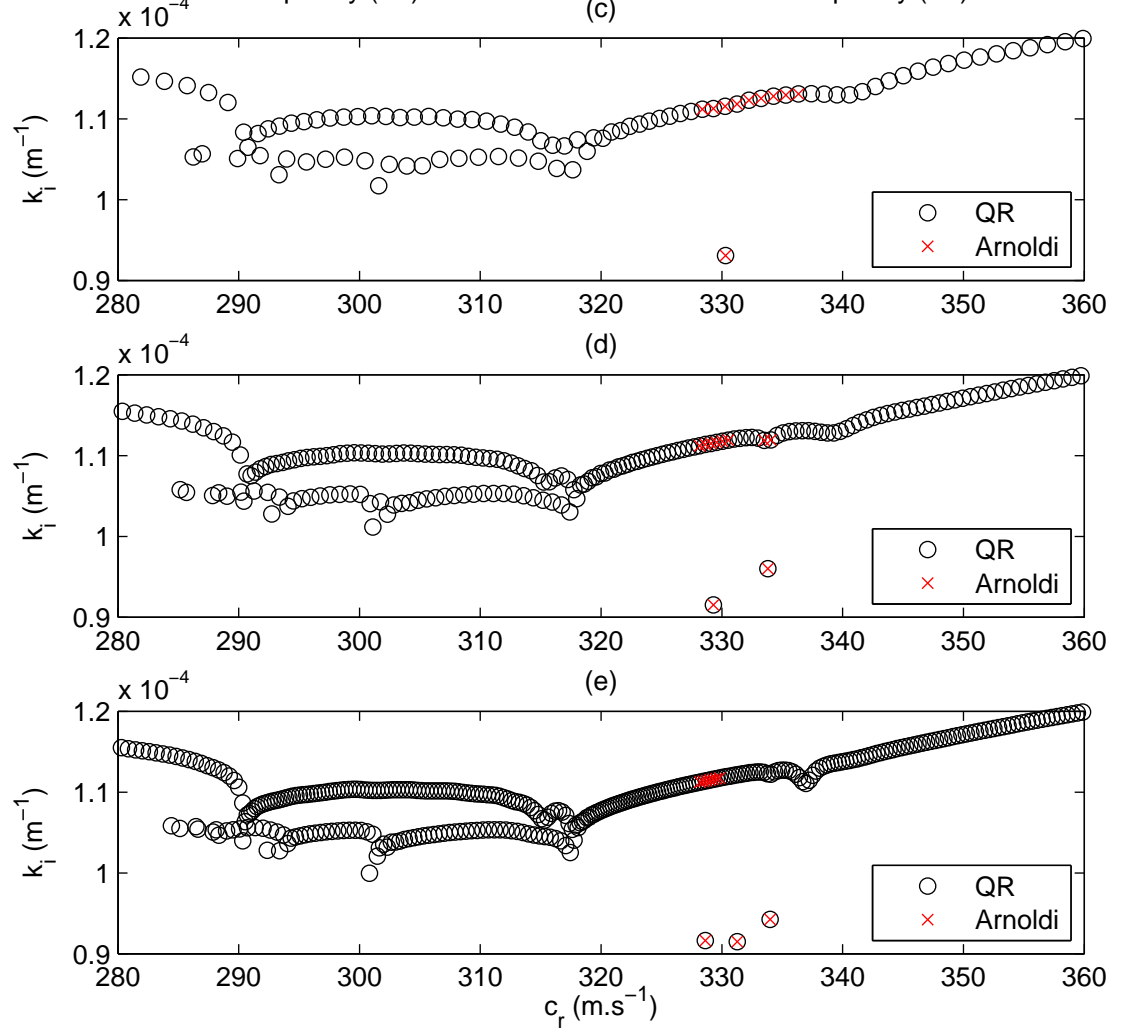

Figure 8: (a) Size $N$ of the matrix discretization for the profile $c_{1 a}(z)$ (figure 1) as a function of the frequency. (b) Computation time for both the QR algorithm (LAPACK) and the implicitly restarted Krylov-Arnoldi algorithm (ARPACK). (c),(d),(e) Eigenvalues computed with both the QR algorithm (whole spectrum) and the Krylov-Arnoldi algorithm (10 Ritz pairs) for frequencies $0.3 \mathrm{~Hz}, 0.6 \mathrm{~Hz}$ and $0.9 \mathrm{~Hz}$, respectively. 
Bertin, Millet and Bouche, J. of the Acoustical Society of America, p. 54
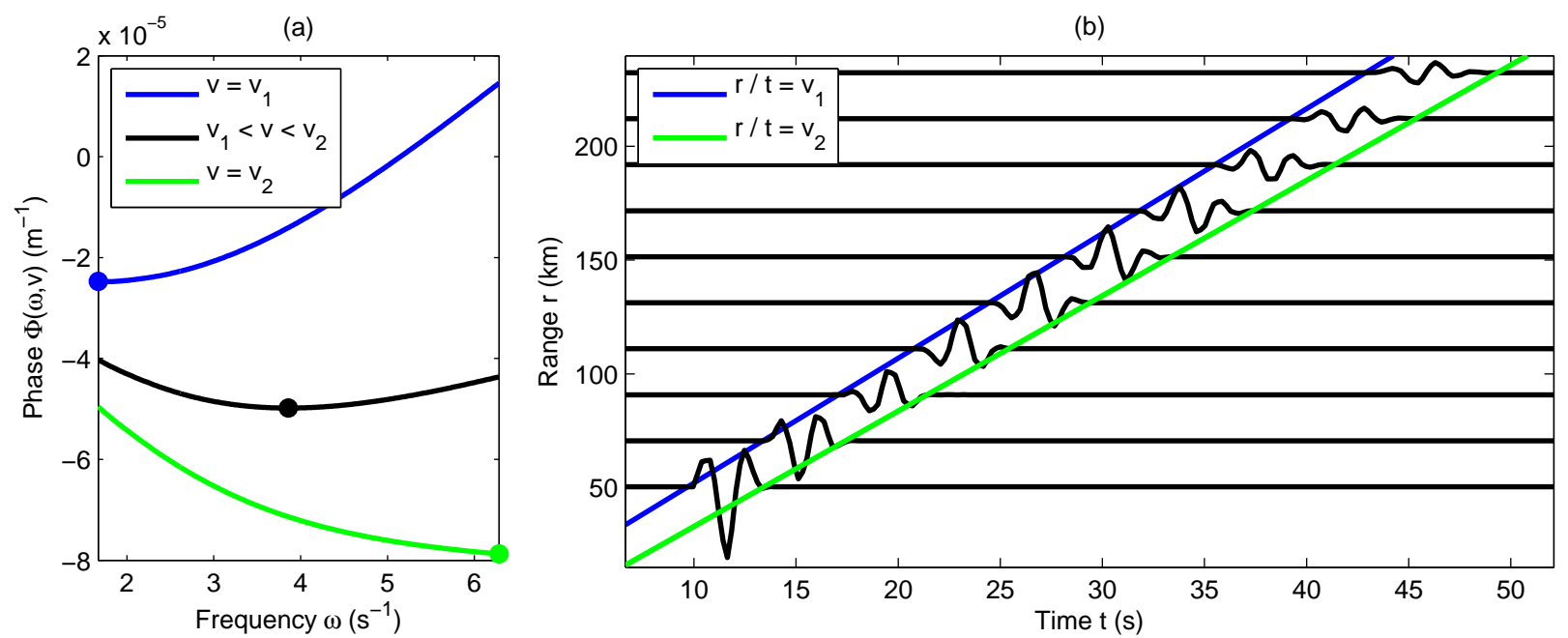

Figure 9: Phase $\Phi$ associated with the first dominant mode as a function of the real frequency $\omega_{r}$ for distinct values of the velocity $v\left(v_{1}=335 \mathrm{~m} \cdot \mathrm{s}^{-1}\right.$ and $\left.v_{2}=328 \mathrm{~m} \cdot \mathrm{s}^{-1}\right)$. Circles show the point of stationary phase $\bar{\omega}$. Ground-based signals obtained with the stationary phase method for the first most sensitive mode. Signals are shown with a shifted time: $t^{\prime}=t-r / c_{0}$ $\left(c_{0}=350 \mathrm{~m} \cdot \mathrm{s}^{-1}\right)$. 
Bertin, Millet and Bouche, J. of the Acoustical Society of America, p. 55

(a)

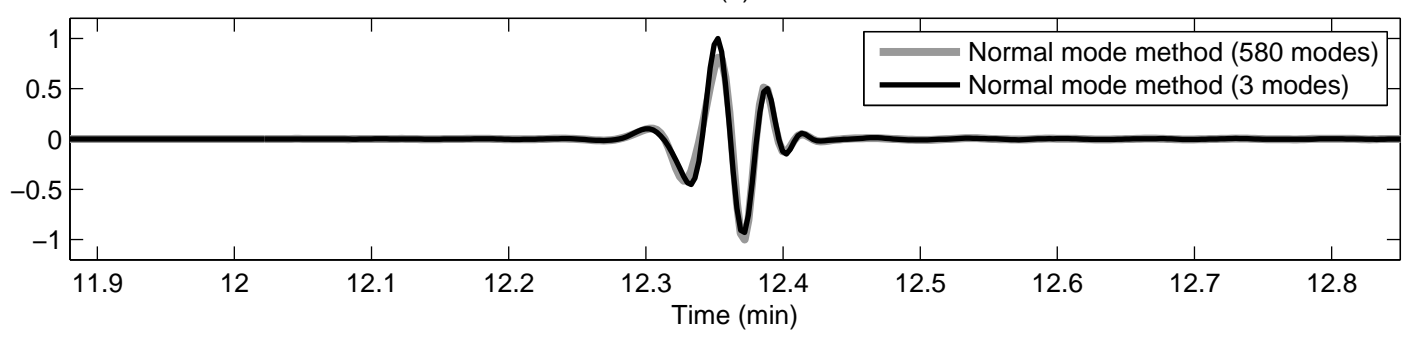

(b)

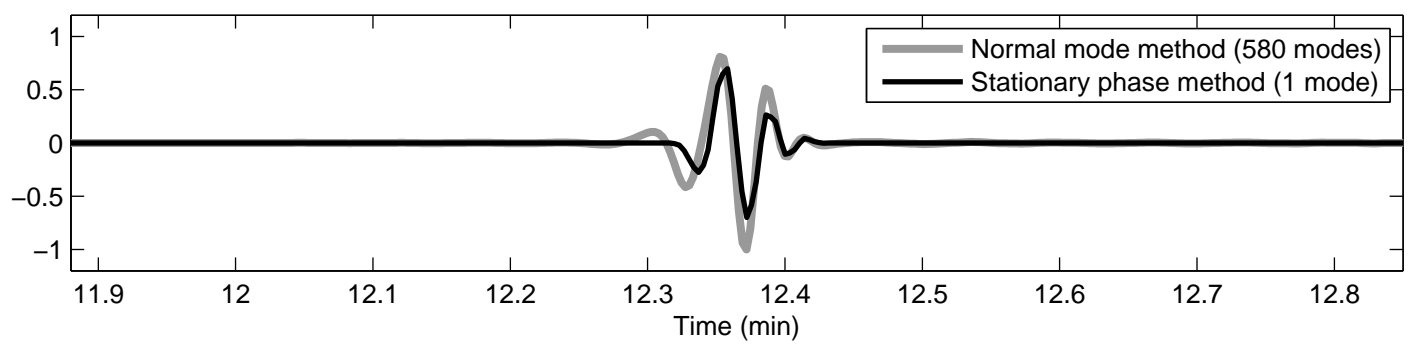

Figure 10: Ground-based signals computed at the station I30JP with the range-dependent normal mode approach. The reference signal (gray), obtained with 580 modes using the FFT algorithm (with 512 frequency samples), is compared to that obtained with the three most sensitive modes (top) and the stationary phase approximation for the first mode (bottom). The source function is given by (20) with a multiplicative constant of $2.65 \times 10^{5}$. 
Bertin, Millet and Bouche, J. of the Acoustical Society of America, p. 56


Figure 11: Ground-based signals (e-g) computed at the station I30JP for three vertical profiles

(a). The profile $c_{2 a}$ (blue) is perturbed by a Gaussian envelope centered at the altitude of maximum effective sound speed. (b-d) Most sensitive eigenvalues lying on $\gamma_{1}$ as $\omega_{r} \rightarrow 2 \pi$ (1 Hz). The eigenvalues obtained with the Krylov-Arnoldi algorithm for $\omega_{r}=2 \pi$ are given by red crosses. 
Bertin, Millet and Bouche, J. of the Acoustical Society of America, p. 57
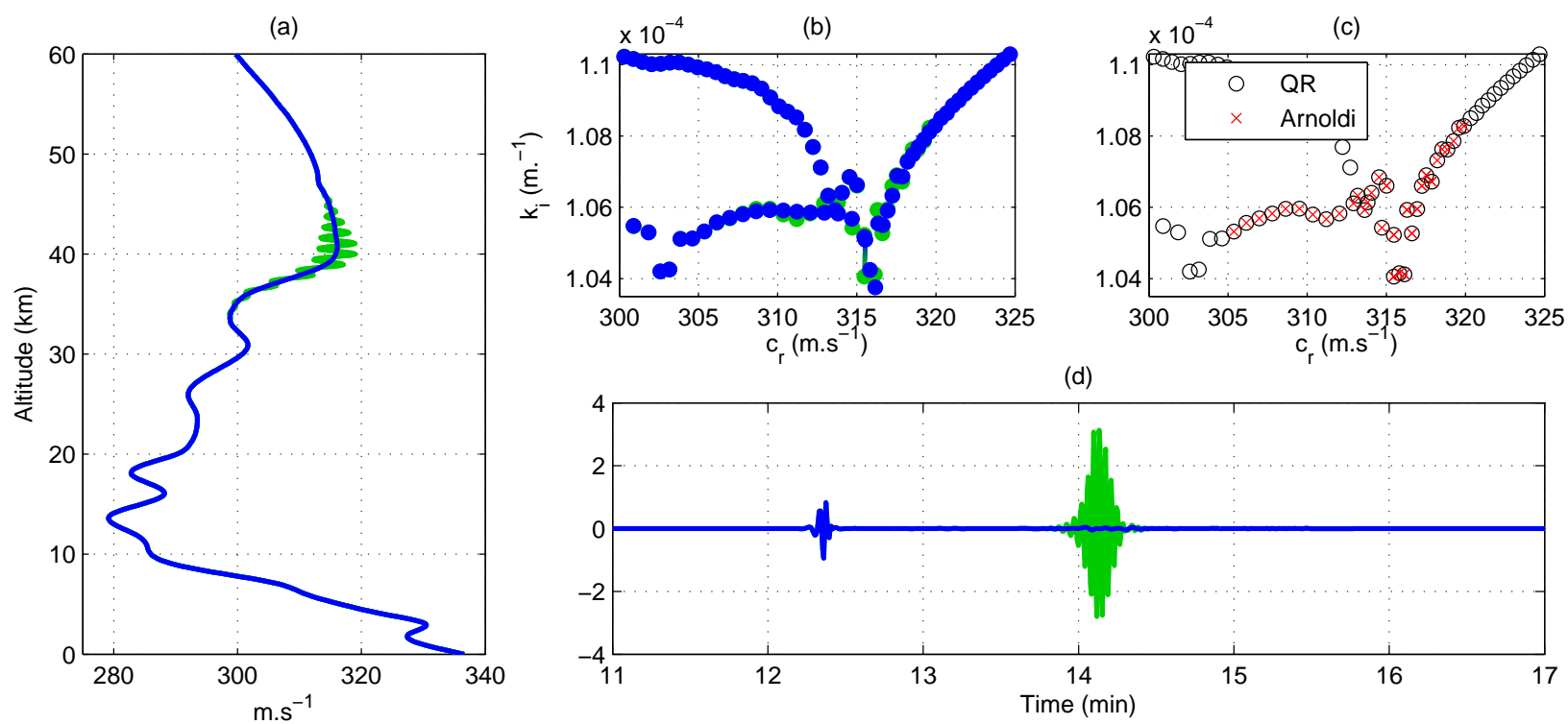

(d)

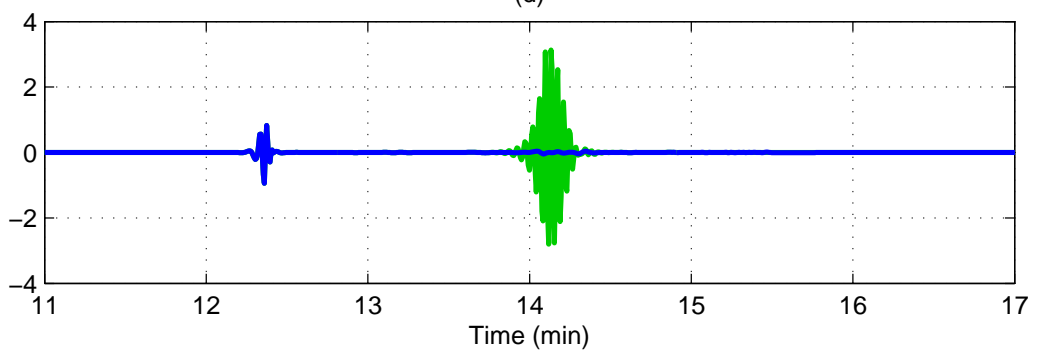

Figure 12: Ground-based signals (d) computed at the station I30JP for two vertical profiles (a). The profile $c_{2 a}$ (blue) is perturbed by a localized upward propagating gravity wave (green profile). The most sensitive eigenvalues (b) are obtained both with a QR algorithm and a Krylov-Arnoldi algorithm (c). The source function is given by (20) with a multiplicative constant of $2.65 \times 10^{5}$. 